\title{
Theory of differential offset continuation ${ }^{a}$
}

${ }^{a}$ Published in Geophysics, 68, 718-732 (2003)

Sergey Fomel

\begin{abstract}
I introduce a partial differential equation to describe the process of prestack reflection data transformation in the offset, midpoint, and time coordinates. The equation is proved theoretically to provide correct kinematics and amplitudes on the transformed constant-offset sections. Solving an initial-value problem with the proposed equation leads to integral and frequency-domain offset continuation operators, which reduce to the known forms of dip moveout operators in the case of continuation to zero offset.
\end{abstract}

\section{INTRODUCTION}

The Earth subsurface is three-dimensional, while seismic reflection data from a multicoverage acquisition belong to a five-dimensional space (time, 2-D offset, and 2-D midpoint coordinates). This fact alone indicates the additional connection that exists in the data space. I show in this paper that it is possible, under certain assumptions, to express this connection in a concise mathematical form of a partial differential equation. The theoretical analysis of this equation allows us to explain and predict the data transformation between different offsets.

The partial differential equation, introduced in this paper $^{1}$, describes the process of offset continuation, which is a transformation of common-offset seismic gathers from one constant offset to another (Bolondi et al., 1982). Bagaini and Spagnolini (1996) identified offset continuation (OC) with a whole family of prestack continuation operators, such as shot continuation (Bagaini and Spagnolini, 1993), dip moveout as a continuation to zero offset (Hale, 1991), and three-dimensional azimuth moveout (Biondi et al., 1998). An intuitive introduction to the concept of offset continuation is presented by Hill et al. (2001). A general data mapping prospective is developed by Bleistein and Jaramillo (2000).

As early as in 1982, Bolondi et al. came up with the idea of describing offset continuation and dip moveout (DMO) as a continuous process by means of a partial differential equation (Bolondi et al., 1982). However, their approximate differential

\footnotetext{
${ }^{1}$ To my knowledge, the first derivation of the revised offset continuation equation was accomplished by Joseph Higginbotham of Texaco in 1989. Unfortunately, Higginbotham's derivation never appeared in the open literature.
} 
operator, built on the results of Deregowski and Rocca's classic paper (Deregowski and Rocca, 1981), failed in the cases of steep reflector dips or large offsets. Hale (1983) writes:

The differences between this algorithm [DMO by Fourier transform] and previously published finite-difference DMO algorithms are analogous to the differences between frequency-wavenumber (Stolt, 1978; Gazdag, 1978) and finite-difference (Claerbout, 1976) algorithms for migration. For example, just as finite-difference migration algorithms require approximations that break down at steep dips, finite-difference DMO algorithms are inaccurate for large offsets and steep dips, even for constant velocity.

Continuing this analogy, we can observe that both finite-difference and frequencydomain migration algorithms share a common origin: the wave equation. The new OC equation, presented in this paper and valid for all offsets and dips, plays a role analogous to that of the wave equation for offset continuation and dip moveout algorithms. A multitude of seismic migration algorithms emerged from the fundamental wave-propagation theory that is embedded in the wave equation. Likewise, the fundamentals of DMO algorithms can be traced to the OC differential equation.

In the first part of the paper, I prove that the revised equation is, under certain assumptions, kinematically valid. This means that wavefronts of the offset continuation process correspond to the reflection wave traveltimes and correctly transform between different offsets. Moreover, the wave amplitudes are also propagated correctly according to the true-amplitude criterion (Black et al., 1993).

In the second part of the paper, I relate the offset continuation equation to different methods of dip moveout. Considering DMO as a continuation to zero offset, I show that DMO operators can be obtained by solving a special initial value problem for the

OC equation. Different known forms of DMO (Hale, 1991) appear as special cases of more general offset continuation operators.

The companion paper (Fomel, 2003b) demonstrates a practical application of differential offset continuation to seismic data interpolation.

\section{INTRODUCING THE OFFSET CONTINUATION EQUATION}

Most of the contents of this paper refer to the following linear partial differential equation:

$$
h\left(\frac{\partial^{2} P}{\partial y^{2}}-\frac{\partial^{2} P}{\partial h^{2}}\right)=t_{n} \frac{\partial^{2} P}{\partial t_{n} \partial h} .
$$

Equation (1) describes an artificial (non-physical) process of transforming reflection seismic data $P\left(y, h, t_{n}\right)$ in the offset-midpoint-time domain. In equation $(1), h$ stands 
for the half-offset $(h=(r-s) / 2$, where $s$ and $r$ are the source and the receiver surface coordinates), $y$ is the midpoint $(y=(r+s) / 2)$, and $t_{n}$ is the time coordinate after normal moveout correction is applied:

$$
t_{n}=\sqrt{t^{2}-\frac{4 h^{2}}{v^{2}}} .
$$

The velocity $v$ is assumed to be known a priori. Equation (1) belongs to the class of linear hyperbolic equations, with the offset $h$ acting as a time-like variable. It describes a wave-like propagation in the offset direction.

\section{Proof of validity}

A simplified version of the ray method technique (Červený, 2001; Babich, 1991) can allow us to prove the theoretical validity of equation (1) for all offsets and reflector dips by deriving two equations that describe separately wavefront (traveltime) and amplitude transformation. According to the formal ray theory, the leading term of the high-frequency asymptotics for a reflected wave recorded on a seismogram takes the form

$$
P\left(y, h, t_{n}\right) \approx A_{n}(y, h) R_{n}\left(t_{n}-\tau_{n}(y, h)\right),
$$

where $A_{n}$ stands for the amplitude, $R_{n}$ is the wavelet shape of the leading highfrequency term, and $\tau_{n}$ is the traveltime curve after normal moveout. Inserting (3) as a trial solution for (1), collecting terms that have the same asymptotic order (correspond to the same-order derivatives of the wavelet $R_{n}$ ), and neglecting loworder terms, we arrive at the set of two first-order partial differential equations:

$$
\begin{gathered}
h\left[\left(\frac{\partial \tau_{n}}{\partial y}\right)^{2}-\left(\frac{\partial \tau_{n}}{\partial h}\right)^{2}\right]=-\tau_{n} \frac{\partial \tau_{n}}{\partial h} \\
\left(\tau_{n}-2 h \frac{\partial \tau_{n}}{\partial h}\right) \frac{\partial A_{n}}{\partial h}+2 h \frac{\partial \tau_{n}}{\partial y} \frac{\partial A_{n}}{\partial y}+h A_{n}\left(\frac{\partial^{2} \tau_{n}}{\partial y^{2}}-\frac{\partial^{2} \tau_{n}}{\partial h^{2}}\right)=0 .
\end{gathered}
$$

Equation (4) describes the transformation of traveltime curve geometry in the OC process analogously to how the eikonal equation describes the front propagation in the classic wave theory. What appear to be wavefronts of the wave motion described by equation (1) are traveltime curves of reflected waves recorded on seismic sections. The law of amplitude transformation for high-frequency wave components related to those wavefronts is given by equation (5). In terms of the theory of partial differential equations, equation (4) is the characteristic equation for (1).

\section{Proof of kinematic equivalence}

In order to prove the validity of equation (4), it is convenient to transform it to the

coordinates of the initial shot gathers: $s=y-h, r=y+h$, and $\tau=\sqrt{\tau_{n}^{2}+\frac{4 h^{2}}{v^{2}}}$. The 
transformed equation takes the form

$$
\left(\tau^{2}+\frac{(r-s)^{2}}{v^{2}}\right)\left(\frac{\partial \tau}{\partial r}-\frac{\partial \tau}{\partial s}\right)=2(r-s) \tau\left(\frac{1}{v^{2}}-\frac{\partial \tau}{\partial r} \frac{\partial \tau}{\partial s}\right) .
$$

Now the goal is to prove that any reflection traveltime function $\tau(r, s)$ in a constant velocity medium satisfies equation (6).

Let $S$ and $R$ be the source and the receiver locations, and $O$ be a reflection point for that pair. Note that the incident ray $S O$ and the reflected ray $O R$ form a triangle with the basis on the offset $S R(l=|S R|=|r-s|)$. Let $\alpha_{1}$ be the angle of $S O$ from the vertical axis, and $\alpha_{2}$ be the analogous angle of $R O$ (Figure 1). The law of sines gives us the following explicit relationships between the sides and the angles of the triangle $S O R$ :

$$
\begin{aligned}
& |S O|=|S R| \frac{\cos \alpha_{2}}{\sin \left(\alpha_{2}-\alpha_{1}\right)}, \\
& |R O|=|S R| \frac{\cos \alpha_{1}}{\sin \left(\alpha_{2}-\alpha_{1}\right)} .
\end{aligned}
$$

Hence, the total length of the reflected ray satisfies

$$
v \tau=|S O|+|R O|=|S R| \frac{\cos \alpha_{1}+\cos \alpha_{2}}{\sin \left(\alpha_{2}-\alpha_{1}\right)}=|r-s| \frac{\cos \alpha}{\sin \gamma} .
$$

Here $\gamma$ is the reflection angle $\left(\gamma=\left(\alpha_{2}-\alpha_{1}\right) / 2\right)$, and $\alpha$ is the central ray angle $\left(\alpha=\left(\alpha_{2}+\alpha_{1}\right) / 2\right)$, which coincides with the local dip angle of the reflector at the reflection point. Recalling the well-known relationships between the ray angles and the first-order traveltime derivatives

$$
\begin{aligned}
& \frac{\partial \tau}{\partial s}=\frac{\sin \alpha_{1}}{v}, \\
& \frac{\partial \tau}{\partial r}=\frac{\sin \alpha_{2}}{v},
\end{aligned}
$$

we can substitute (9), (10), and (11) into (6), which leads to the simple trigonometric equality

$$
\cos ^{2}\left(\frac{\alpha_{1}+\alpha_{2}}{2}\right)+\sin ^{2}\left(\frac{\alpha_{1}-\alpha_{2}}{2}\right)=1-\sin \alpha_{1} \sin \alpha_{2} .
$$

It is now easy to show that equality (12) is true for any $\alpha_{1}$ and $\alpha_{2}$, since

$$
\sin ^{2} a-\sin ^{2} b=\sin (a+b) \sin (a-b) .
$$

Thus we have proved that equation (6), equivalent to (4), is valid in constant velocity media independently of the reflector geometry and the offset. This means that high-frequency asymptotic components of the waves, described by the OC equation, are located on the true reflection traveltime curves.

The theory of characteristics can provide other ways to prove the kinematic validity of equation (4), as described by Fomel (1994) and Goldin (1994). 
Figure 1: Reflection rays in a constant velocity medium (a scheme).

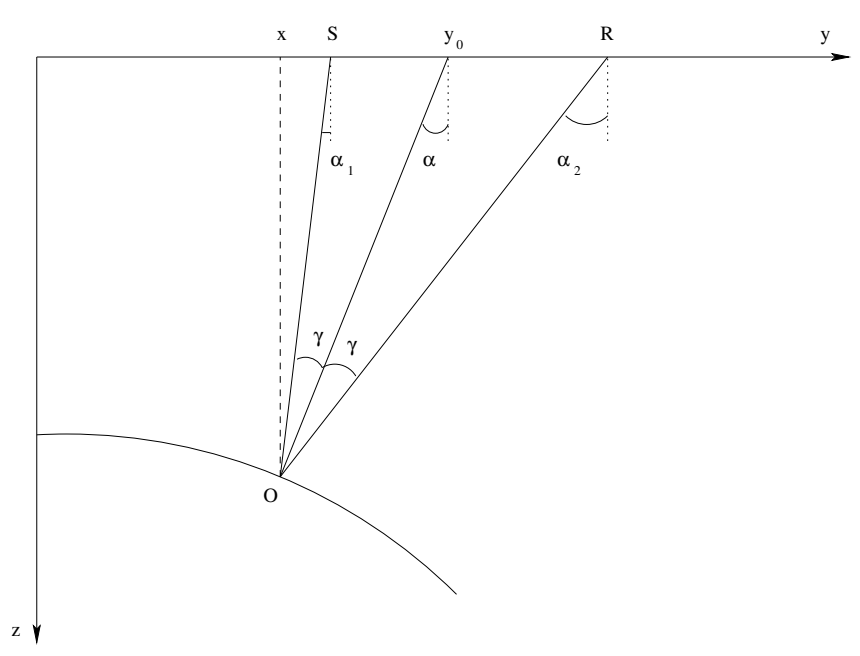

\section{Comparison with Bolondi's OC equation}

Equation (1) and the previously published OC equation (Bolondi et al., 1982) differ only with respect to the single term $\frac{\partial^{2} P}{\partial h^{2}}$. However, this difference is substantial.

From the offset continuation characteristic equation (4), we can conclude that the first-order traveltime derivative with respect to offset decreases with decreasing offset. The derivative equals zero at the zero offset, as predicted by the principle of reciprocity (the reflection traveltime has to be an even function of offset). Neglecting $\left(\frac{\partial \tau_{n}}{\partial h}\right)^{2}$ in (4) leads to the characteristic equation

$$
h\left(\frac{\partial \tau_{n}}{\partial y}\right)^{2}=-\tau_{n} \frac{\partial \tau_{n}}{\partial h}
$$

which corresponds to the approximate OC equation of Bolondi et al. (1982). The approximate equation has the form

$$
h \frac{\partial^{2} P}{\partial y^{2}}=t_{n} \frac{\partial^{2} P}{\partial t_{n} \partial h} .
$$

Comparing equations (13) and (4), we can note that approximation (13) is valid only if

$$
\left(\frac{\partial \tau_{n}}{\partial h}\right)^{2} \ll\left(\frac{\partial \tau_{n}}{\partial y}\right)^{2}
$$

To find the geometric constraints implied by inequality (15), we can express the traveltime derivatives in geometric terms. As follows from expressions (10) and (11),

$$
\begin{aligned}
& \frac{\partial \tau}{\partial y}=\frac{\partial \tau}{\partial r}+\frac{\partial \tau}{\partial s}=\frac{2 \sin \alpha \cos \gamma}{v} \\
& \frac{\partial \tau}{\partial h}=\frac{\partial \tau}{\partial r}-\frac{\partial \tau}{\partial s}=\frac{2 \cos \alpha \sin \gamma}{v}
\end{aligned}
$$


Expression (9) allows transforming equations (16) and (17) to the form

$$
\begin{aligned}
\tau_{n} \frac{\partial \tau_{n}}{\partial y} & =\tau \frac{\partial \tau}{\partial y}=4 h \frac{\sin \alpha \cos \alpha \cot \gamma}{v^{2}} \\
\tau_{n} \frac{\partial \tau_{n}}{\partial h} & =\tau \frac{\partial \tau}{\partial h}-\frac{4 h}{v^{2}}=-4 h \frac{\sin ^{2} \alpha}{v^{2}}
\end{aligned}
$$

Without loss of generality, we can assume $\alpha$ to be positive. Consider a plane tangent to a true reflector at the reflection point (Figure 2). The traveltime of a wave, reflected from the plane, has the known explicit expression

$$
\tau=\frac{2}{v} \sqrt{L^{2}+h^{2} \cos ^{2} \alpha}
$$

where $L$ is the length of the normal ray from the midpoint. As follows from combining (20) and (9),

$$
\cos \alpha \cot \gamma=\frac{L}{h}
$$

We can now combine equations (21), (18), and (19) to transform inequality (15) to the form

$$
h \ll \frac{L}{\sin \alpha}=z \cot \alpha,
$$

where $z$ is the depth of the plane reflector under the midpoint. For example, for a dip of 45 degrees, equation (14) will be satisfied only for offsets that are much smaller than the depth of the reflector.

Figure 2: Reflection rays and tangent to the reflector in a constant velocity medium (a scheme).

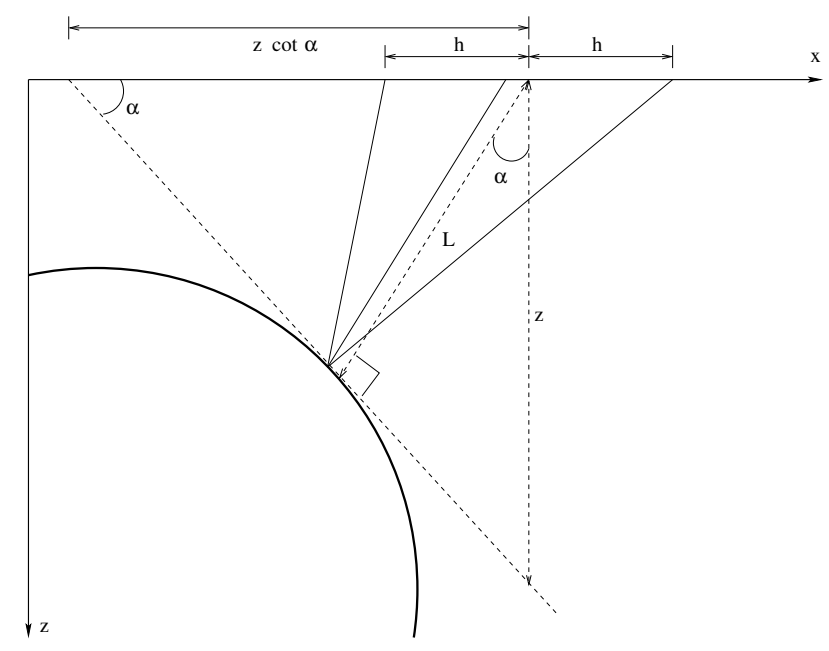

\section{Offset continuation geometry: time rays}

To study the laws of traveltime curve transformation in the OC process, it is convenient to apply the method of characteristics (Courant, 1962) to the eikonal-type equation (4). The characteristics of equation (4) [bi-characteristics with respect to equation (1)] are the trajectories of the high-frequency energy propagation in the 
imaginary OC process. Following the formal analogy with seismic rays, I call those trajectories time rays, where the word time refers to the fact that the trajectories describe the traveltime transformation (Fomel, 1994). According to the theory of first-order partial differential equations, time rays are determined by a set of ordinary differential equations (characteristic equations) derived from equation (4) :

$$
\begin{array}{r}
\frac{d y}{d t_{n}}=-\frac{2 h Y}{t_{n} H}, \frac{d Y}{d t_{n}}=\frac{Y}{t_{n}}, \\
\frac{d h}{d t_{n}}=-\frac{1}{H}+\frac{2 h}{t_{n}}, \frac{d H}{d t_{n}}=\frac{Y^{2}}{t_{n} H},
\end{array}
$$

where $Y$ corresponds to $\frac{\partial \tau_{n}}{\partial y}$ along a ray and $H$ corresponds to $\frac{\partial \tau_{n}}{\partial h}$. In this notation, equation (4) takes the form

$$
h\left(Y^{2}-H^{2}\right)=-t_{n} H
$$

and serves as an additional constraint for the definition of time rays. System (23) can be solved by standard mathematical methods (Tenenbaum and Pollard, 1985). Its general solution takes the parametric form, where the time variable $t_{n}$ is the parameter changing along a time ray:

$$
\begin{gathered}
y\left(t_{n}\right)=C_{1}-C_{2} t_{n}^{2} \quad ; \quad h\left(t_{n}\right)=t_{n} \sqrt{C_{2}^{2} t_{n}^{2}+C_{3}} ; \\
Y\left(t_{n}\right)=\frac{C_{2} t_{n}}{C_{3}} \quad ; \quad H\left(t_{n}\right)=\frac{h}{C_{3} t_{n}}
\end{gathered}
$$

and $C_{1}, C_{2}$, and $C_{3}$ are independent coefficients, constant along each time ray. To find the values of these coefficients, we can pose an initial-value problem for the system of differential equations (23). The traveltime curve $\tau_{n}(y ; h)$ for a given common offset $h$ and the first partial derivative $\frac{\partial \tau_{n}}{\partial h}$ along the same constant offset section provide natural initial conditions. A particular case of those conditions is the zero-offset traveltime curve. If the first partial derivative of traveltime with respect to offset is continuous, it vanishes at zero offset according to the reciprocity principle (traveltime must be an even function of the offset): $t_{0}\left(y_{0}\right)=\tau_{n}(y ; 0),\left.\frac{\partial \tau_{n}}{\partial h}\right|_{h=0}=0$. Applying the initial-value conditions to the general solution (26) generates the following expressions for the ray invariants:

$$
\begin{aligned}
C_{1} & =y+h \frac{Y}{H}=y_{0}-\frac{t_{0}\left(y_{0}\right)}{t_{0}^{\prime}\left(y_{0}\right)} ; C_{2}=\frac{h Y}{\tau_{n}^{2} H}=-\frac{1}{t_{0}\left(y_{0}\right) t_{0}^{\prime}\left(y_{0}\right)} ; \\
C_{3} & =\frac{h}{\tau_{n} H}=-\frac{1}{\left(t_{0}^{\prime}\left(y_{0}\right)\right)^{2}},
\end{aligned}
$$

where $t_{0}^{\prime}\left(y_{0}\right)$ denotes the derivative $\frac{d t_{0}}{d y_{0}}$. Finally, substituting equations (27) into (26), we obtain an explicit parametric form of the ray trajectories:

$$
\begin{aligned}
y_{1}\left(t_{1}\right) & =y+\frac{h Y}{t_{n}^{2} H}\left(t_{n}^{2}-t_{1}^{2}\right)=y_{0}+\frac{t_{1}^{2}-t_{0}^{2}\left(y_{0}\right)}{t_{0}\left(y_{0}\right) t_{0}^{\prime}\left(y_{0}\right)} \\
h_{1}^{2}\left(t_{1}\right) & =\frac{h t_{1}^{2}}{t_{n}^{3} H}\left(t_{n}^{2}+t_{1}^{2} \frac{h Y^{2}}{t_{n} H}\right)=t_{1}^{2} \frac{t_{1}^{2}-t_{0}^{2}\left(y_{0}\right)}{\left(t_{0}\left(y_{0}\right) t_{0}^{\prime}\left(y_{0}\right)\right)^{2}} .
\end{aligned}
$$


Here $y_{1}, h_{1}$, and $t_{1}$ are the coordinates of the continued seismic section. Equations (28) indicates that the time ray projections to a common-offset section have a parabolic form. Time rays do not exist for $t_{0}^{\prime}\left(y_{0}\right)=0$ (a locally horizontal reflector) because in this case post-NMO offset continuation transform is not required.

The actual parameter that determines a particular time ray is the reflection point location. This important conclusion follows from the known parametric equations

$$
\begin{aligned}
& t_{0}(x)=t_{v} \sec \alpha=t_{v}(x) \sqrt{1+u^{2}\left(t_{v}^{\prime}(x)\right)^{2}}, \\
& y_{0}(x)=x+u t_{v} \tan \alpha=x+u^{2} t_{v}(x) t_{v}^{\prime}(x),
\end{aligned}
$$

where $x$ is the reflection point, $u$ is half of the wave velocity $(u=v / 2), t_{v}$ is the vertical time (reflector depth divided by $u$ ), and $\alpha$ is the local reflector dip. Taking into account that the derivative of the zero-offset traveltime curve is

$$
\frac{d t_{0}}{d y_{0}}=\frac{t_{0}^{\prime}(x)}{y_{0}^{\prime}(x)}=\frac{\sin \alpha}{u}=\frac{t_{v}^{\prime}(x)}{\sqrt{1+u^{2}\left(t_{v}^{\prime}(x)\right)^{2}}}
$$

and substituting equations (30) and (31) into (28) and (29), we get

$$
\begin{aligned}
y_{1}\left(t_{1}\right) & =x+\frac{t_{1}^{2}-t_{v}^{2}(x)}{t_{v}(x) t_{v}^{\prime}(x)} \\
u^{2} t^{2}\left(t_{1}\right) & =t_{1}^{2} \frac{t_{1}^{2}-t_{v}^{2}(x)}{\left(t_{v}(x) t_{v}^{\prime}(x)\right)^{2}}
\end{aligned}
$$

where $t^{2}\left(t_{1}\right)=t_{1}^{2}+h_{1}^{2}\left(t_{1}\right) / u^{2}$.

To visualize the concept of time rays, let us consider some simple analytic examples of its application to geometric analysis of the offset-continuation process.

\section{Example 1: plane reflector}

The simplest and most important example is the case of a plane dipping reflector. Putting the origin of the $y$ axis at the intersection of the reflector plane with the surface, we can express the reflection traveltime after NMO in the form

$$
\tau_{n}(y, h)=p \sqrt{y^{2}-h^{2}},
$$

where $p=2 \frac{\sin \alpha}{v}$, and $\alpha$ is the dip angle. The zero-offset traveltime in this case is a straight line:

$$
t_{0}\left(y_{0}\right)=p y_{0} .
$$

According to equations (28-29), the time rays in this case are defined by

$$
y_{1}\left(t_{1}\right)=\frac{t_{1}^{2}}{p^{2} y_{0}} ; h_{1}^{2}\left(t_{1}\right)=t_{1}^{2} \frac{t_{1}^{2}-p^{2} y_{0}^{2}}{p^{4} y_{0}^{2}} ; y_{0}=\frac{y^{2}-h^{2}}{y} \text {. }
$$

The geometry of the OC transformation is shown in Figure 3. 

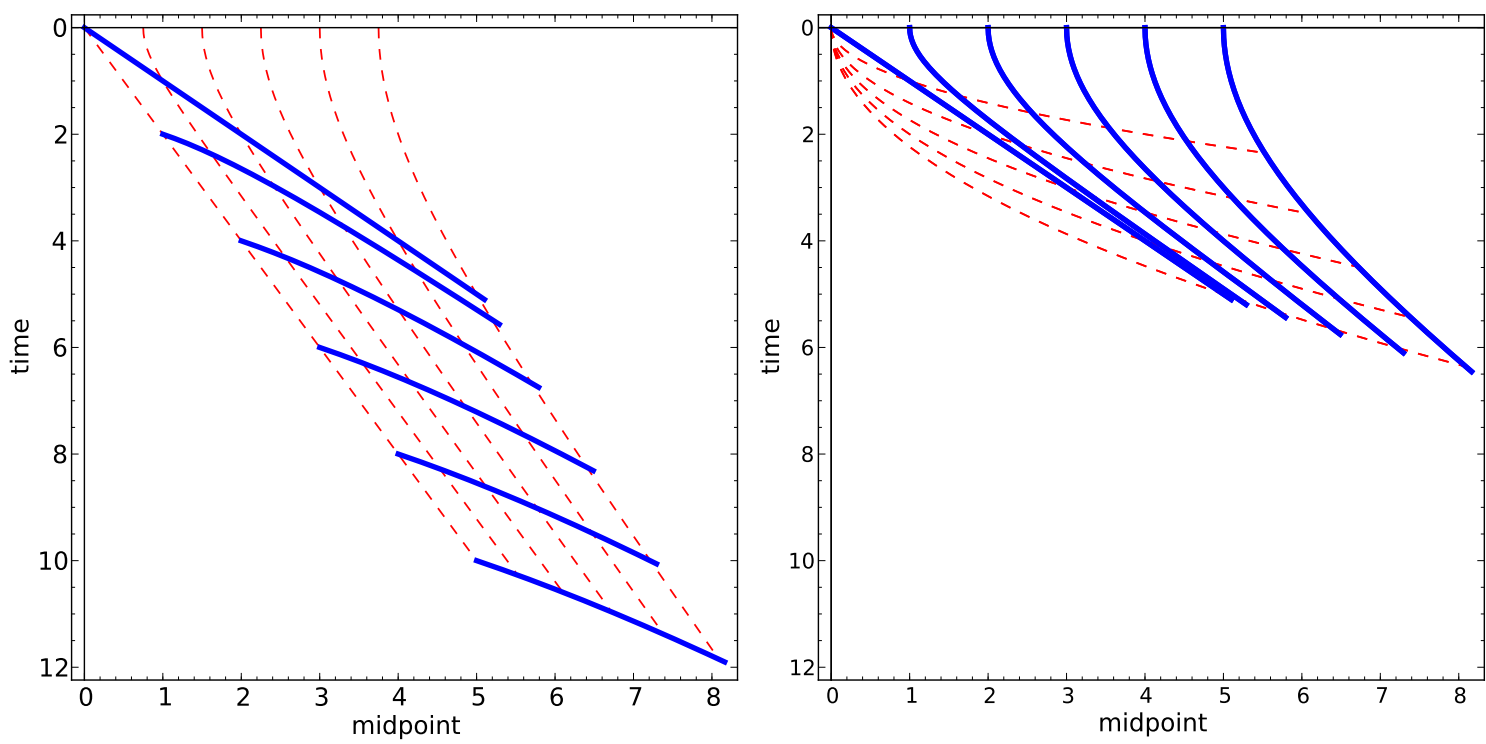

Figure 3: Transformation of the reflection traveltime curves in the OC process: the case of a plane dipping reflector. Left: Time coordinate before the NMO correction. Right: Time coordinate after NMO. The solid lines indicate traveltime curves at different common-offset sections; the dashed lines indicate time rays.

\section{Example 2: point diffractor}

The second example is the case of a point diffractor (the left side of Figure 4). Without loss of generality, the origin of the midpoint axis can be put above the diffraction point. In this case the zero-offset reflection traveltime curve has the well-known hyperbolic form

$$
t_{0}\left(y_{0}\right)=\frac{\sqrt{z^{2}+y_{0}^{2}}}{u}
$$

where $z$ is the depth of the diffractor and $u=v / 2$ is half of the wave velocity. Time rays are defined according to equations (28-29), as follows:

$$
y_{1}\left(t_{1}\right)=\frac{u^{2} t_{1}^{2}-z^{2}}{y_{0}} ; u^{2} t^{2}\left(t_{1}\right)=u^{2} t_{1}^{2}+h_{1}^{2}\left(t_{1}\right)=u^{2} t_{1}^{2} \frac{u^{2} t_{1}^{2}-z^{2}}{y_{0}^{2}} .
$$

\section{Example 3: elliptic reflector}

The third example (the right side of Figure 4) is the curious case of a focusing elliptic reflector. Let $y$ be the center of the ellipse and $h$ be half the distance between the foci of the ellipse. If both foci are on the surface, the zero-offset traveltime curve is defined by the so-called "DMO smile" (Deregowski and Rocca, 1981):

$$
t_{0}\left(y_{0}\right)=\frac{t_{n}}{h} \sqrt{h^{2}-\left(y-y_{0}\right)^{2}},
$$



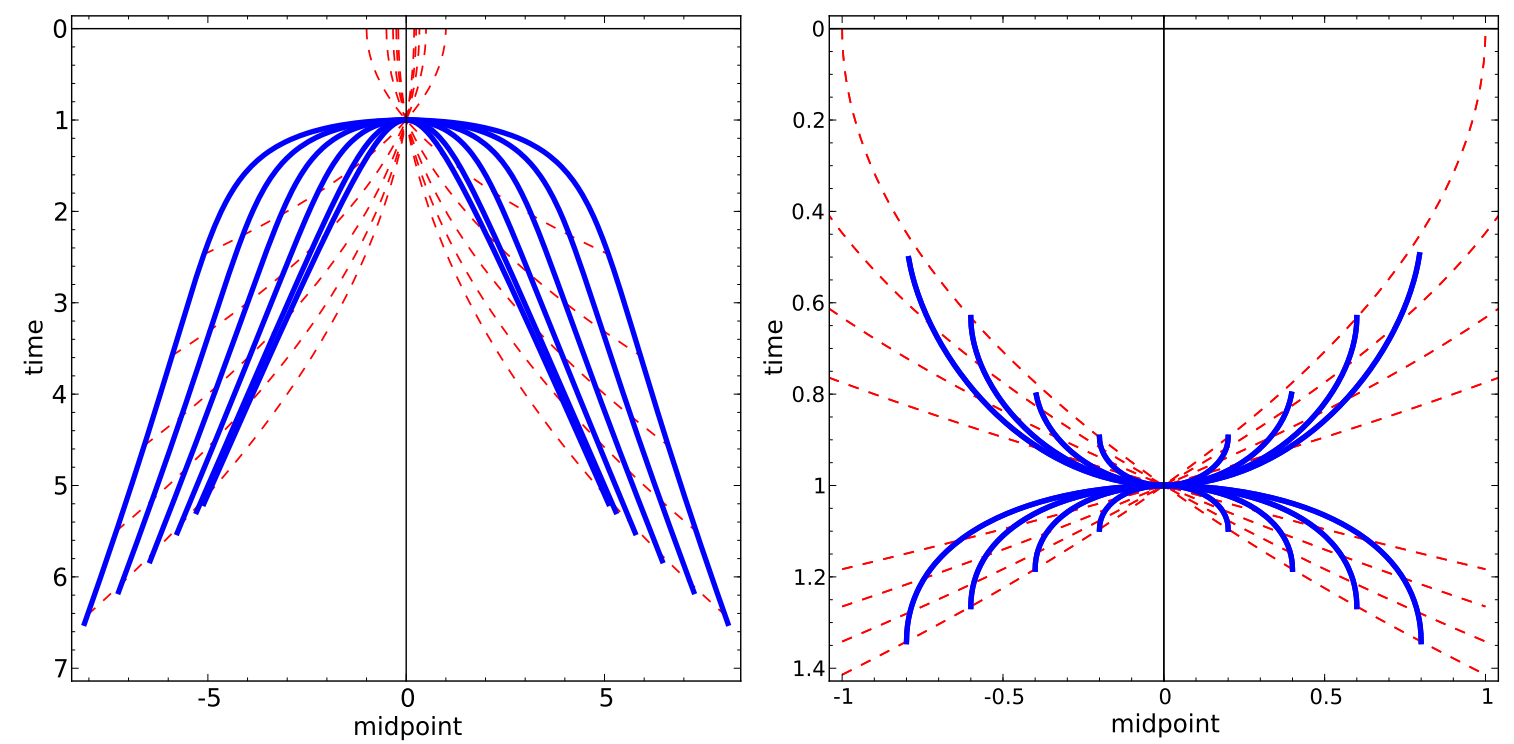

Figure 4: Transformation of the reflection traveltime curves in the OC process. Left: the case of a diffraction point. Right: the case of an elliptic reflector. Solid lines indicate traveltime curves at different common-offset sections, dashed lines indicate time rays.

where $t_{n}=2 z / v$, and $z$ is the small semi-axis of the ellipse. The time-ray equations are

$$
y_{1}\left(t_{1}\right)=y+\frac{h^{2}}{y-y_{0}} \frac{t_{1}^{2}-t_{n}^{2}}{t_{n}^{2}} ; h_{1}^{2}\left(t_{1}\right)=h^{2} \frac{t_{1}^{2}}{t_{n}^{2}}\left(1+\frac{h^{2}}{\left(y-y_{0}\right)^{2}} \frac{t_{1}^{2}-t_{n}^{2}}{t_{n}^{2}}\right) .
$$

When $y_{1}$ coincides with $y$, and $h_{1}$ coincides with $h$, the source and the receiver are in the foci of the elliptic reflector, and the traveltime curve degenerates to a point $t_{1}=t_{n}$. This remarkable fact is the actual basis of the geometric theory of dip moveout (Deregowski and Rocca, 1981).

\section{Proof of amplitude equivalence}

Let us now consider the connection between the laws of traveltime transformation and the laws of the corresponding amplitude transformation. The change of the wave amplitudes in the OC process is described by the first-order partial differential transport equation (5). We can find the general solution of this equation by applying the method of characteristics. The solution takes the explicit integral form

$$
A_{n}\left(t_{n}\right)=A_{0}\left(t_{0}\right) \exp \left(\int_{t_{o}}^{t_{n}}\left[h\left(\frac{\partial^{2} \tau_{n}}{\partial y^{2}}-\frac{\partial^{2} \tau_{n}}{\partial h^{2}}\right)\left(\tau_{n} \frac{\partial \tau_{n}}{\partial h}\right)^{-1}\right] d \tau_{n}\right) .
$$

The integral in equation (42) is defined on a curved time ray, and $A_{n}\left(t_{n}\right)$ stands for the amplitude transported along this ray. In the case of a plane dipping reflector, the ray 
amplitude can be immediately evaluated by substituting the explicit traveltime and time ray equations from the preceding section into (42). The amplitude expression in this case takes the simple form

$$
A_{n}\left(t_{n}\right)=A_{0}\left(t_{0}\right) \exp \left(-\int_{t_{o}}^{t_{n}} \frac{d \tau_{n}}{\tau_{n}}\right)=A_{0}\left(t_{0}\right) \frac{t_{0}}{t_{n}} .
$$

In order to consider the more general case of a curvilinear reflector, we need to take into account the connection between the traveltime derivatives in (42) and the geometry of the reflector. As follows directly from the trigonometry of the incident and reflected rays triangle (Figure 1),

$$
\begin{aligned}
h & =\frac{r-s}{2}=D \frac{\cos \alpha \sin \gamma \cos \gamma}{\cos ^{2} \alpha-\sin ^{2} \gamma}, \\
y & =\frac{r+s}{2}=x+D \frac{\cos ^{2} \alpha \sin \alpha}{\cos ^{2} \alpha-\sin ^{2} \gamma}, \\
y_{0} & =x+D \sin \alpha,
\end{aligned}
$$

where $D$ is the length of the normal ray. Let $\tau_{0}=2 D / v$ be the zero-offset reflection traveltime. Combining equations (44) and (46) with (9), we can get the following relationship:

$$
a=\frac{\tau_{n}}{\tau_{0}}=\frac{\cos \alpha \cos \gamma}{\left(\cos ^{2} \alpha-\sin ^{2} \gamma\right)^{1 / 2}}=\left(1+\frac{\sin ^{2} \alpha \sin ^{2} \gamma}{\cos ^{2} \alpha-\sin ^{2} \gamma}\right)^{1 / 2}=\frac{h}{\sqrt{h^{2}-\left(y-y_{0}\right)^{2}}},
$$

which describes the "DMO smile" (40) found by Deregowski and Rocca (1981) in geometric terms. Equation (47) allows for a convenient change of variables in equation (42). Let the reflection angle $\gamma$ be a parameter monotonically increasing along a time ray. In this case, each time ray is uniquely determined by the position of the reflection point, which in turn is defined by the values of $D$ and $\alpha$. According to this change of variables, we can differentiate (47) along a time ray to get

$$
\frac{d \tau_{n}}{\tau_{n}}=-\frac{\sin ^{2} \alpha}{2 \cos ^{2} \gamma\left(\cos ^{2} \gamma-\sin ^{2} \alpha\right)} d\left(\cos ^{2} \gamma\right)
$$

Note also that the quantity $h\left(\tau_{n} \frac{\partial \tau_{n}}{\partial h}\right)^{-1}$ in equation (42) coincides exactly with the time ray invariant $C_{3}$ found in equation (27). Therefore its value is constant along each time ray and equals

$$
h\left(\tau_{n} \frac{\partial \tau_{n}}{\partial h}\right)^{-1}=-\frac{v^{2}}{4 \sin ^{2} \alpha} .
$$

Finally, as shown in Appendix,

$$
\tau_{n}\left(\frac{\partial^{2} \tau_{n}}{\partial y^{2}}-\frac{\partial^{2} \tau_{n}}{\partial h^{2}}\right)=4 \frac{\cos ^{2} \gamma}{v^{2}}\left(\frac{\sin ^{2} \alpha+D K}{\cos ^{2} \gamma+D K}\right),
$$


where $K$ is the reflector curvature at the reflection point. Substituting (48), (49), and (50) into (42) transforms the integral to the form

$$
\begin{gathered}
\int_{t_{o}}^{t_{n}}\left[h\left(\frac{\partial^{2} \tau_{n}}{\partial y^{2}}-\frac{\partial^{2} \tau_{n}}{\partial h^{2}}\right)\left(\tau_{n} \frac{\partial \tau_{n}}{\partial h}\right)^{-1}\right] d \tau_{n}= \\
=-\frac{1}{2} \int_{\cos ^{2} \gamma_{0}}^{\cos ^{2} \gamma}\left(\frac{1}{\cos ^{2} \gamma^{\prime}-\sin ^{2} \alpha}-\frac{1}{\cos ^{2} \gamma^{\prime}+D K}\right) d\left(\cos ^{2} \gamma^{\prime}\right)
\end{gathered}
$$

which we can evaluate analytically. The final equation for the amplitude transformation is

$$
\begin{aligned}
A_{n} & =A_{0} \frac{\sqrt{\cos ^{2} \gamma-\sin ^{2} \alpha}}{\sqrt{\cos ^{2} \gamma_{0}-\sin ^{2} \alpha}}\left(\frac{\cos ^{2} \gamma_{0}+D K}{\cos ^{2} \gamma+D K}\right)^{1 / 2}= \\
& =A_{0} \frac{\tau_{0} \cos \gamma}{\tau_{n} \cos \gamma_{0}}\left(\frac{\cos ^{2} \gamma_{0}+D K}{\cos ^{2} \gamma+D K}\right)^{1 / 2} .
\end{aligned}
$$

In case of a plane reflector, the curvature $K$ is zero, and equation (52) coincides with (43). In the general case can be rewritten as

$$
A_{n}=\frac{c \cos \gamma}{\tau_{n} \sqrt{\cos ^{2} \gamma+D K}}
$$

where $c$ is constant along each time ray (it may vary with the reflection point location on the reflector but not with the offset). We should compare equation (53) with the known expression for the reflection wave amplitude of the leading ray series term in 2.5-D media (Bleistein et al., 2001):

$$
A=\frac{C_{R}(\gamma) \Psi}{G}
$$

where $C_{R}$ stands for the angle-dependent reflection coefficient, $G$ is the geometric spreading

$$
G=v \tau \frac{\sqrt{\cos ^{2} \gamma+D K}}{\cos \gamma}
$$

and $\Psi$ includes other possible factors (such as the source directivity) that we can either correct or neglect in the preliminary processing. It is evident that the curvature dependence of the amplitude transformation (53) coincides completely with the true geometric spreading factor (55) and that the angle dependence of the reflection coefficient is not accounted for the offset continuation process. If the wavelet shape of the reflected wave on seismic sections $\left[R_{n}\right.$ in equation (3) $]$ is described by the delta function, then, as follows from the known properties of this function,

$$
A \delta(t-\tau(y, h))=\left|\frac{d t_{n}}{d t}\right| A \delta\left(t_{n}-\tau_{n}(y, h)\right)=\frac{t}{t_{n}} A \delta\left(t_{n}-\tau_{n}(y, h)\right)
$$


which leads to the equality

$$
A_{n}=A \frac{t}{t_{n}} .
$$

Combining equation (57) with equations (54) and (53) allows us to evaluate the amplitude after continuation from some initial offset $h_{0}$ to another offset $h_{1}$, as follows:

$$
A_{1}=\frac{C_{R}\left(\gamma_{0}\right) \Psi_{0}}{G_{1}}
$$

According to equation (58), the OC process described by equation (1) is amplitudepreserving in the sense that corresponds to the definition of Born DMO (Bleistein, 1990; Liner, 1991). This means that the geometric spreading factor from the initial amplitudes is transformed to the true geometric spreading on the continued section, while the reflection coefficient stays the same. This remarkable dynamic property allows AVO (amplitude versus offset) analysis to be performed by a dynamic comparison between true constant-offset sections and the sections transformed by OC from different offsets. With a simple trick, the offset coordinate is transferred to the reflection angles for the AVO analysis. As follows from (47) and (9),

$$
\frac{\tau_{n}^{2}}{\tau \tau_{0}}=\cos \gamma
$$

If we include the $\frac{t_{n}^{2}}{t t_{0}}$ factor in the DMO operator (continuation to zero offset) and divide the result by the DMO section obtained without this factor, the resultant amplitude of the reflected events will be directly proportional to $\cos \gamma$, where the reflection angle $\gamma$ corresponds to the initial offset. Of course, this conclusion is rigorously valid for constant-velocity 2.5 -D media only.

Black et al. (1993) suggest a definition of true-amplitude DMO different from that of Born DMO. The difference consists of two important components:

1. True-amplitude DMO addresses preserving the peak amplitude of the image wavelet instead of preserving its spectral density. In the terms of this paper, the peak amplitude corresponds to the pre-NMO amplitude $A$ from formula (54) instead of corresponding to the spectral density amplitude $A_{n}$. A simple correction factor $\frac{t}{t_{n}}$ would help us take the difference between the two amplitudes into account. Multiplication by $\frac{t}{t_{n}}$ can be easily done at the NMO stage.

2. Seismic sections are multiplied by time to correct for the geometric spreading factor prior to DMO (or, in our case, offset continuation) processing.

As follows from (55), multiplication by $t$ is a valid geometric spreading correction for plane reflectors only. It is the amplitude-preserving offset continuation based on the $\mathrm{OC}$ equation (1) that is able to correct for the curvature-dependent factor in the amplitude. To take into account the second aspect of Black's definition, we can consider the modified field $\hat{P}$ such that

$$
\hat{P}\left(y, h, t_{n}\right)=t P\left(y, h, t_{n}\right) .
$$


Substituting (60) into the OC equation (1) transforms the latter to the form

$$
h\left(\frac{\partial^{2} \hat{P}}{\partial y^{2}}-\frac{\partial^{2} \hat{P}}{\partial h^{2}}\right)=t_{n} \frac{\partial^{2} \hat{P}}{\partial t_{n} \partial h}-\frac{\partial \hat{P}}{\partial h} .
$$

Equations (61) and (1) differ only with respect to the first-order damping term $\frac{\partial \hat{P}}{\partial h}$. This term affects the amplitude behavior but not the traveltimes, since the eikonaltype equation (4) depends on the second-order terms only. Offset continuation operators based on (61) conform to Black's definition of true-amplitude processing.

Fomel and Bleistein (2001) describe an alternative approach to confirming the kinematic and amplitude validity of the offset continuation equation. Applying equation (1) directly on the Kirchhoff model of prestack seismic data shows that the equation is satisfied to the same asymptotic order of accuracy as the Kirchhoff modeling approximation (Haddon and Buchen, 1981; Bleistein, 1984).

\section{INTEGRAL OFFSET CONTINUATION OPERATOR}

Equation (1) describes a continuous process of reflected wavefield continuation in the time-offset-midpoint domain. In order to find an integral-type operator that performs the one-step offset continuation, I consider the following initial-value problem for equation (1):

Given a post-NMO constant-offset section at half-offset $h_{1}$

$$
\left.P\left(t_{n}, h, y\right)\right|_{h=h_{1}}=P_{1}^{(0)}\left(t_{n}, y\right)
$$

and its first-order derivative with respect to offset

$$
\left.\frac{\partial P\left(t_{n}, h, y\right)}{\partial h}\right|_{h=h_{1}}=P_{1}^{(1)}\left(t_{n}, y\right),
$$

find the corresponding section $P^{(0)}\left(t_{n}, y\right)$ at offset $h$.

Equation (1) belongs to the hyperbolic type, with the offset coordinate $h$ being a "time-like" variable and the midpoint coordinate $y$ and the time $t_{n}$ being "space-like" variables. The last condition (63) is required for the initial value problem to be wellposed (Courant, 1962). From a physical point of view, its role is to separate the two different wave-like processes embedded in equation (1), which are analogous to inward and outward wave propagation. We will associate the first process with continuation to a larger offset and the second one with continuation to a smaller offset. Though the offset derivatives of data are not measured in practice, they can be estimated from the data at neighboring offsets by a finite-difference approximation. Selecting a propagation branch explicitly, for example by considering the high-frequency asymptotics of the continuation operators, can allow us to eliminate the need for condition (63). In this section, I discuss the exact integral solution of the OC equation and analyze its asymptotics. 
The integral solution of problem (62-63) for equation (1) is obtained in with the help of the classic methods of mathematical physics (Fomel, 1994, 2001). It takes the explicit form

$$
\begin{aligned}
P\left(t_{n}, h, y\right) & =\iint P_{1}^{(0)}\left(t_{1}, y_{1}\right) G_{0}\left(t_{1}, h_{1}, y_{1} ; t_{n}, h, y\right) d t_{1} d y_{1} \\
+ & \iint P_{1}^{(1)}\left(t_{1}, y_{1}\right) G_{1}\left(t_{1}, h_{1}, y_{1} ; t_{n}, h, y\right) d t_{1} d y_{1},
\end{aligned}
$$

where the Green's functions $G_{0}$ and $G_{1}$ are expressed as

$$
\begin{aligned}
& G_{0}\left(t_{1}, h_{1}, y_{1} ; t_{n}, h, y\right)=\operatorname{sign}\left(h-h_{1}\right) \frac{H\left(t_{n}\right)}{\pi} \frac{\partial}{\partial t_{n}}\left\{\frac{H(\Theta)}{\sqrt{\Theta}}\right\}, \\
& G_{1}\left(t_{1}, h_{1}, y_{1} ; t_{n}, h, y\right)=\operatorname{sign}\left(h-h_{1}\right) \frac{H\left(t_{n}\right)}{\pi} h \frac{t_{n}}{t_{1}^{2}}\left\{\frac{H(\Theta)}{\sqrt{\Theta}}\right\},
\end{aligned}
$$

and the parameter $\Theta$ is

$$
\Theta\left(t_{1}, h_{1}, y_{1} ; t_{n}, h, y\right)=\left(h_{1}^{2} / t_{1}^{2}-h^{2} / t_{n}^{2}\right)\left(t_{1}^{2}-t_{n}^{2}\right)-\left(y_{1}-y\right)^{2} .
$$

$H$ stands for the Heaviside step-function.

From equations (65) and (66) one can see that the impulse response of the offset continuation operator is discontinuous in the time-offset-midpoint space on a surface defined by the equality

$$
\Theta\left(t_{1}, h_{1}, y_{1} ; t_{n}, h, y\right)=0,
$$

which describes the "wavefronts" of the offset continuation process. In terms of the theory of characteristics (Courant, 1962), the surface $\Theta=0$ corresponds to the characteristic conoid formed by the bi-characteristics of equation (1) - time rays emerging from the point $\left\{t_{n}, h, y\right\}=\left\{t_{1}, h_{1}, y_{1}\right\}$. The common-offset slices of the characteristic conoid are shown in the left plot of Figure 5.

As a second-order differential equation of the hyperbolic type, equation (1) describes two different processes. The first process is "forward" continuation from smaller to larger offsets, the second one is "reverse" continuation in the opposite direction. These two processes are clearly separated in the high-frequency asymptotics of operator (64). To obtain the asymptotic representation, it is sufficient to note that $\frac{1}{\sqrt{\pi}} \frac{H(t)}{\sqrt{t}}$ is the impulse response of the causal half-order integration operator and that $\frac{H\left(t^{2}-a^{2}\right)}{\sqrt{t^{2}-a^{2}}}$ is asymptotically equivalent to $\frac{H(t-a)}{\sqrt{2 a} \sqrt{t-a}}(t, a>0)$. Thus, the asymptotical form of the integral offset-continuation operator becomes

$$
\begin{aligned}
P^{( \pm)}\left(t_{n}, h, y\right) & =\mathbf{D}_{ \pm t_{n}}^{1 / 2} \int w_{0}^{( \pm)}\left(\xi ; h_{1}, h, t_{n}\right) P_{1}^{(0)}\left(\theta^{( \pm)}\left(\xi ; h_{1}, h, t_{n}\right), y_{1}-\xi\right) d \xi \\
& \pm \mathbf{I}_{ \pm t_{n}}^{1 / 2} \int w_{1}^{( \pm)}\left(\xi ; h_{1}, h, t_{n}\right) P_{1}^{(1)}\left(\theta^{( \pm)}\left(\xi ; h_{1}, h, t_{n}\right), y_{1}-\xi\right) d \xi
\end{aligned}
$$

Here the signs "+" and "-" correspond to the type of continuation (the sign of $\left.h-h_{1}\right), \mathbf{D}_{ \pm t_{n}}^{1 / 2}$ and $\mathbf{I}_{ \pm t_{n}}^{1 / 2}$ stand for the operators of causal and anticausal half-order 

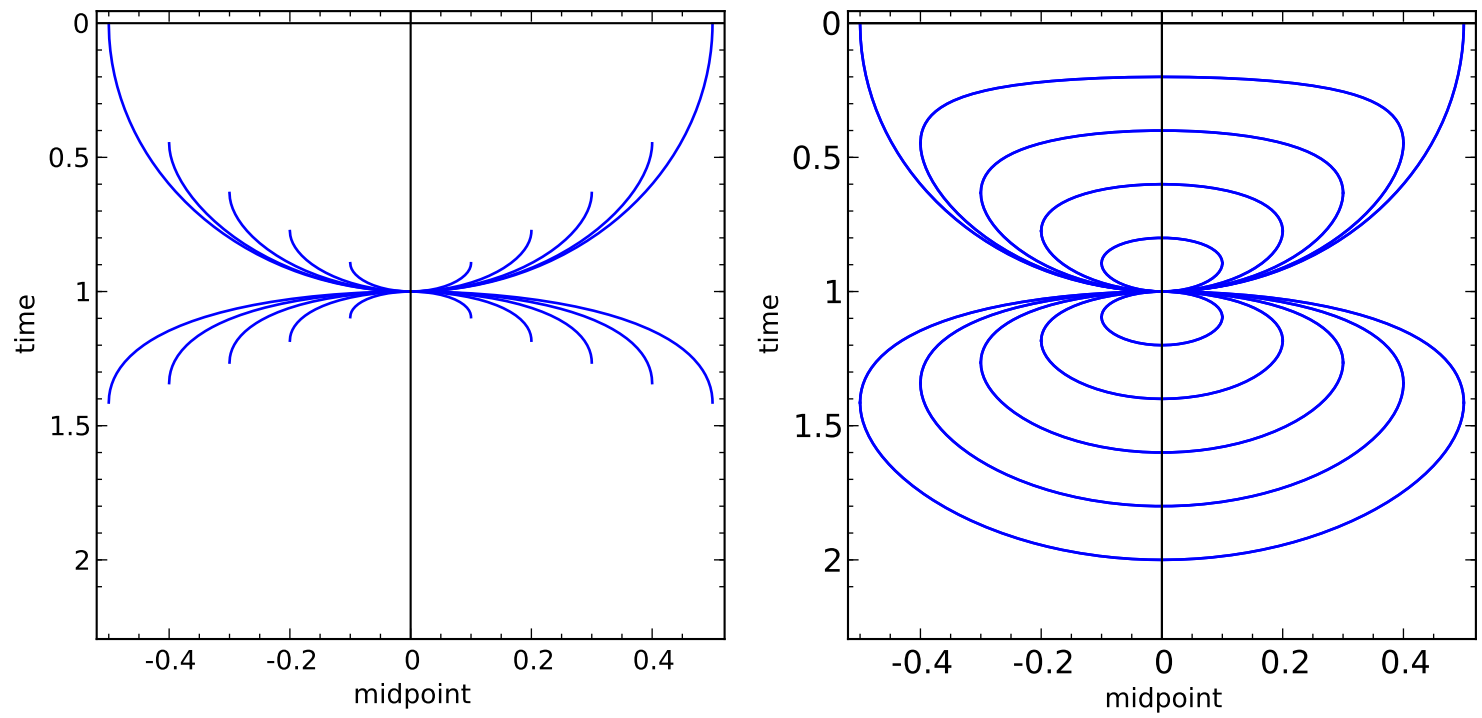

Figure 5: Constant-offset sections of the characteristic conoid - "offset continuation fronts" (left), and branches of the conoid used in the integral OC operator (right). The upper part of the plots (small times) corresponds to continuation to smaller offsets; the lower part (large times) corresponds to larger offsets.

differentiation and integration applied with respect to the time variable $t_{n}$, the summation paths $\theta^{( \pm)}\left(\xi ; h_{1}, h, t_{n}\right)$ correspond to the two non-negative sections of the characteristic conoid (68) (Figure 5):

$$
t_{1}=\theta^{( \pm)}\left(\xi ; h_{1}, h, t_{n}\right)=\frac{t_{n}}{h} \sqrt{\frac{U \pm V}{2}},
$$

where $U=h^{2}+h_{1}^{2}-\xi^{2}$, and $V=\sqrt{U^{2}-4 h^{2} h_{1}^{2}} ; \xi$ is the midpoint separation (the integration parameter), and $w_{0}^{( \pm)}$and $w_{1}^{( \pm)}$are the following weighting functions:

$$
\begin{aligned}
w_{0}^{( \pm)} & =\frac{1}{\sqrt{2 \pi}} \frac{\theta^{( \pm)}\left(\xi ; h_{1}, h, t_{n}\right)}{\sqrt{t_{n} V}}, \\
w_{1}^{( \pm)} & =\frac{1}{\sqrt{2 \pi}} \frac{\sqrt{t_{n}} h_{1}}{\sqrt{V} \theta^{( \pm)}\left(\xi ; h_{1}, h, t_{n}\right)} .
\end{aligned}
$$

Expression (70) for the summation path of the OC operator was obtained previously by Stovas and Fomel (1996) and Biondi and Chemingui (1994). A somewhat different form of it is proposed by Bagaini and Spagnolini (1996). I describe the kinematic interpretation of formula (70) in Appendix B.

In the high-frequency asymptotics, it is possible to replace the two terms in equation (69) with a single term (Fomel, 2003a). The single-term expression is

$$
P^{( \pm)}\left(t_{n}, h, y\right)=\mathbf{D}_{ \pm t_{n}}^{1 / 2} \int w^{( \pm)}\left(\xi ; h_{1}, h, t_{n}\right) P_{1}^{(0)}\left(\theta^{( \pm)}\left(\xi ; h_{1}, h, t_{n}\right), y_{1}-\xi\right) d \xi
$$


where

$$
\begin{aligned}
w^{(+)} & =\sqrt{\frac{\theta^{(+)}\left(\xi ; h_{1}, h, t_{n}\right)}{2 \pi}} \frac{h^{2}-h_{1}^{2}-\xi^{2}}{V^{3 / 2}}, \\
w^{(-)} & =\frac{\theta^{(-)}\left(\xi ; h_{1}, h, t_{n}\right)}{\sqrt{2 \pi t_{n}}} \frac{h_{1}^{2}-h^{2}+\xi^{2}}{V^{3 / 2}} .
\end{aligned}
$$

A more general approach to true-amplitude asymptotic offset continuation is developed by ?.

The limit of expression (70) for the output offset $h$ approaching zero can be evaluated by L'Hospitale's rule. As one would expect, it coincides with the well-known expression for the summation path of the integral DMO operator (Deregowski and Rocca, 1981)

$$
t_{1}=\theta^{(-)}\left(\xi ; h_{1}, 0, t_{n}\right)=\lim _{h \rightarrow 0} \frac{t_{n}}{h} \sqrt{\frac{U-V}{2}}=\frac{t_{n} h_{1}}{\sqrt{h_{1}^{2}-\xi^{2}}}
$$

I discuss the connection between offset continuation and DMO in the next section.

\section{OFFSET CONTINUATION AND DMO}

Dip moveout represents a particular case of offset continuation for the output offset

equal to zero. In this section, I consider the DMO case separately in order to compare the solutions of equation (1) with the Fourier-domain DMO operators, which have been the standard for DMO processing since Hale's outstanding work (Hale, 1983, 1984).

Equation (64) transforms to the time-wavenumber domain with the help of integral tables:

$$
\widetilde{P}\left(t_{n}, h, k\right)=H\left(t_{n}\right)\left(\widetilde{P}_{0}\left(t_{n}, h, k\right)+t_{n} \widetilde{P}_{1}\left(t_{n}, h, k\right)\right),
$$

where

$$
\begin{gathered}
\widetilde{P}_{0}=\frac{\partial}{\partial t_{n}} \int_{\left(h_{1} / h\right) t_{n}}^{t_{n}} \widetilde{P}_{1}^{(0)}\left(\left|t_{1}\right|, k\right) J_{0}\left(k \sqrt{\left(\frac{h^{2}}{t_{n}^{2}}-\frac{h_{1}^{2}}{t_{1}^{2}}\right)\left(t_{n}^{2}-t_{1}^{2}\right)}\right) d t_{1} \\
\widetilde{P}_{1}=\int_{\left(h_{1} / h\right) t_{n}}^{t_{n}} h_{1} \widetilde{P}_{1}^{(1)}\left(\left|t_{1}\right|, k\right) J_{0}\left(k \sqrt{\left(\frac{h^{2}}{t_{n}^{2}}-\frac{h_{1}^{2}}{t_{1}^{2}}\right)\left(t_{n}^{2}-t_{1}^{2}\right)}\right) \frac{d t_{1}}{t_{1}^{2}} \\
\widetilde{P}_{1}^{(j)}\left(t_{1}, k\right)=\int P_{1}^{(j)}\left(t_{1}, y_{1}\right) \exp \left(-i k y_{1}\right) d y_{1}(j=0,1) \\
\widetilde{P}\left(t_{n}, h, k\right)=\int P\left(t_{n}, h, y\right) \exp (-i k y) d y(j=0,1) .
\end{gathered}
$$


Setting the output offset to zero, we obtain the following DMO-like integral operators in the $t-k$ domain:

$$
\widetilde{P}\left(t_{0}, 0, k\right)=H\left(t_{0}\right)\left(\widetilde{P}_{0}\left(t_{0}, k\right)+t_{0} \widetilde{P}_{1}\left(t_{0}, k\right)\right)
$$

where

$$
\begin{aligned}
& \widetilde{P}_{0}\left(t_{0}, k\right)=-\frac{\partial}{\partial t_{0}} \int_{t_{0}}^{\infty} \widetilde{P}_{1}^{(0)}\left(\left|t_{1}\right|, k\right) J_{0}\left(\frac{k h_{1}}{t_{1}} \sqrt{t_{1}^{2}-t_{0}^{2}}\right) d t_{1} \\
& \widetilde{P}_{1}\left(t_{0}, k\right)=-\int_{t_{0}}^{\infty} h_{1} \widetilde{P}_{1}^{(1)}\left(\left|t_{1}\right|, k\right) J_{0}\left(\frac{k h_{1}}{t_{1}} \sqrt{t_{1}^{2}-t_{0}^{2}}\right) \frac{d t_{1}}{t_{1}^{2}}
\end{aligned}
$$

the wavenumber $k$ corresponds to the midpoint axis $y$, and $J_{0}$ is the zeroth-order Bessel function. The Fourier transform of (83) and (84) with respect to the time variable $t_{0}$ reduces to known integrals (Gradshtein and Ryzhik, 1994) and creates explicit DMO-type operators in the frequency-wavenumber domain, as follows:

$$
\begin{gathered}
\widetilde{\widetilde{P}}_{0}\left(\omega_{0}, k\right)=i \int_{-\infty}^{\infty} \widetilde{P}_{1}^{(0)}\left(\left|t_{1}\right|, k\right) \frac{\sin \left(\omega_{0}\left|t_{1}\right| A\right)}{A} d t_{1}, \\
\widetilde{\widetilde{P}}_{1}\left(\omega_{0}, k\right)=i \int_{-\infty}^{\infty} h_{1} \widetilde{P}_{1}^{(1)}\left(\left|t_{1}\right|, k\right) \frac{\sin \left(\omega_{0}\left|t_{1}\right| A\right)}{A} \frac{d t_{1}}{t_{1}^{2}},
\end{gathered}
$$

where

$$
\begin{gathered}
A=\sqrt{1+\frac{\left(k h_{1}\right)^{2}}{\left(\omega_{0} t_{1}\right)^{2}}}, \\
\widetilde{\widetilde{P}}_{j}\left(\omega_{0}, k\right)=\int \widetilde{P}_{j}\left(t_{0}, k\right) \exp \left(i \omega_{0} t_{0}\right) d t_{0} .
\end{gathered}
$$

It is interesting to note that the first term of the continuation to zero offset (85) coincides exactly with the imaginary part of Hale's DMO operator (Hale, 1984). However, unlike Hale's, operator (82) is causal, which means that its impulse response does not continue to negative times. The non-causality of Hale's DMO and related issues are discussed in more detail by Stovas and Fomel (1996).

Though Hale's DMO is known to provide correct reconstruction of the geometry of zero-offset reflections, it does not account properly for the amplitude changes (Black et al., 1993). The preceding section of this paper shows that the additional contribution to the amplitude is contained in the second term of the OC operator (64), which transforms to the second term in the DMO operator (82). Note that this term vanishes at the input offset equal to zero, which represents the case of the inverse DMO operator.

Considering the inverse DMO operator as the continuation from zero offset to a non-zero offset, we can obtain its representation in the $t-k$ domain from equations (7779) as

$$
\widetilde{P}\left(t_{n}, h, k\right)=H\left(t_{n}\right) \frac{\partial}{\partial t_{n}} \int_{0}^{t_{n}} \widetilde{P}_{0}\left(\left|t_{0}\right|, k\right) J_{0}\left(\frac{k h}{t_{n}} \sqrt{t_{n}^{2}-t_{0}^{2}}\right) d t_{0}
$$


Fourier transforming equation (89) with respect to the time variable $t_{0}$ according to equation (88), we get the Fourier-domain version of the "amplitude-preserving" inverse DMO:

$$
\begin{gathered}
\widetilde{P}\left(t_{n}, h, k\right)=\frac{H\left(t_{n}\right)}{2 \pi} \frac{\partial}{\partial t_{n}} \int_{-\infty}^{\infty} \widetilde{\widetilde{P}}_{0}\left(\omega_{0}, k\right) \frac{\sin \left(\omega_{0}\left|t_{n}\right| A\right)}{\omega_{0} A} d \omega_{0} \\
A=\sqrt{1+\frac{(k h)^{2}}{\left(\omega_{0} t_{n}\right)^{2}}} .
\end{gathered}
$$

Comparing operator (90) with Ronen's version of inverse DMO (Ronen, 1987), one can see that if Hale's DMO is denoted by $\mathbf{D}_{t_{0}} \mathbf{H}$, then Ronen's inverse DMO is $\mathbf{H}^{\mathbf{T}} \mathbf{D}_{-t_{0}}$, while the amplitude-preserving inverse $(90)$ is $\mathbf{D}_{t_{n}} \mathbf{H}^{\mathbf{T}}$. Here $\mathbf{D}_{t}$ is the derivative operator $\left(\frac{\partial}{\partial t}\right)$, and $\mathbf{H}^{\mathbf{T}}$ stands for the adjoint operator defined by the dotproduct test

$$
(\mathbf{H m}, \mathbf{d})=\left(\mathbf{m}, \mathbf{H}^{\mathbf{T}} \mathbf{d}\right),
$$

where the parentheses denote the dot product:

$$
\left(\mathbf{m}_{\mathbf{1}}, \mathbf{m}_{\mathbf{2}}\right)=\iint m_{1}\left(t_{n}, y\right) m_{2}\left(t_{n}, y\right) d t_{n} d y
$$

In high-frequency asymptotics, the difference between the amplitudes of the two inverses is simply the Jacobian term $\frac{d t_{0}}{d t_{n}}$, asymptotically equal to $\frac{t_{0}}{t_{n}}$. This difference corresponds exactly to the difference between Black's definition of amplitude preservation (Black et al., 1993) and the definition used in Born DMO (Bleistein, 1990; Liner, 1991), as discussed above. While operator (90) preserves amplitudes in the Born DMO sense, Ronen's inverse satisfies Black's amplitude preservation criteria. This means Ronen's operator implies that the "geometric spreading" correction (multiplication by time) has been performed on the data prior to DMO.

To construct a one-term DMO operator, thus avoiding the estimation of the offset derivative in (72), let us consider the problem of inverting the inverse DMO operator (90). One of the possible approaches to this problem is the least-squares iterative inversion, as proposed by Ronen (1987). This requires constructing the adjoint operator, which is Hale's DMO (or its analog) in the case of Ronen's method. The iterative least-squares approach can account for irregularities in the data geometry (Ronen et al., 1991; Ronen, 1994) and boundary effects, but it is computationally expensive because of the multiple application of the operators. An alternative approach is the asymptotic inversion, which can be viewed as a special case of preconditioning the adjoint operator (Liner and Cohen, 1988; Chemingui and Biondi, 1996). The goal of the asymptotic inverse is to reconstruct the geometry and the amplitudes of the reflection events in the high-frequency asymptotic limit.

According to Beylkin's theory of asymptotic inversion, also known as the generalized Radon transform (Beylkin, 1985), two operators of the form

$$
D(\omega)=\int X(t, \omega) M(t) \exp [i \omega \phi(t, \omega)] d t
$$


and

$$
\widetilde{M}(t)=\int Y(t, \omega) D(\omega) \exp [-i \omega \phi(t, \omega)] d \omega
$$

constitute a pair of asymptotically inverse operators $(\widetilde{M}(t)$ matching $M(t)$ in the high-frequency asymptotics) if

$$
X(t, \omega) Y(t, \omega)=\frac{Z(t, \omega)}{2 \pi},
$$

where $Z$ is the "Beylkin determinant"

$$
Z(t, \omega)=\left|\frac{\partial \omega}{\partial \hat{\omega}}\right| \text { for } \hat{\omega}=\omega \frac{\partial \phi(t, \omega)}{\partial t} .
$$

With respect to the high-frequency asymptotic representation, we can recast (90) in the equivalent form by moving the time derivative under the integral sign:

$$
\widetilde{P}\left(t_{n}, k\right) \approx \frac{H\left(t_{n}\right)}{2 \pi} \operatorname{Re}\left[\int_{-\infty}^{\infty} A^{-2} \widetilde{\widetilde{P}}_{0}\left(\omega_{0}, k\right) \exp \left(-i \omega_{0}\left|t_{n}\right| A\right) d \omega_{0}\right]
$$

Now the asymptotic inverse of (98) is evaluated by means of Beylkin's method (94)(95), which leads to an amplitude-preserving one-term DMO operator of the form

$$
\widetilde{\widetilde{P}}_{0}\left(\omega_{0}, k\right)=\operatorname{Im}\left[\int_{-\infty}^{\infty} B \widetilde{P}_{1}^{(0)}\left(\left|t_{1}\right|, k\right) \exp \left(i \omega_{0}\left|t_{1}\right| A\right) d t_{1}\right],
$$

where

$$
B=A^{2} \frac{\partial}{\partial \omega_{0}}\left(\omega_{0} \frac{\partial\left(t_{n} A\right)}{\partial t_{n}}\right)=A^{-1}\left(2 A^{2}-1\right) .
$$

The amplitude factor (100) corresponds exactly to that of Born DMO (Bleistein, 1990) in full accordance with the conclusions of the asymptotic analysis of the offsetcontinuation amplitudes. An analogous result can be obtained with the different definition of amplitude preservation proposed by Black et al. (1993). In the time-andspace domain, the operator asymptotically analogous to (99) is found by applying either the stationary phase technique (Liner, 1990; Black et al., 1993) or Goldin's method of discontinuities (Goldin, 1988, 1990), which is the time-and-space analog of Beylkin's asymptotic inverse theory (Stovas and Fomel, 1996). The time-and-space asymptotic DMO operator takes the form

$$
P_{0}\left(t_{0}, y\right)=\mathbf{D}_{-t_{0}}^{1 / 2} \int w_{0}\left(\xi ; h_{1}, t_{0}\right) P_{1}^{(0)}\left(\theta^{(-)}\left(\xi ; h_{1}, 0, t_{0}\right), y_{1}-\xi\right) d \xi,
$$

where the weighting function $w_{0}$ is defined as

$$
w_{0}\left(\xi ; h_{1}, t_{0}\right)=\sqrt{\frac{t_{0}}{2 \pi}} \frac{h_{1}\left(h_{1}^{2}+\xi^{2}\right)}{\left(h_{1}^{2}-\xi^{2}\right)^{2}} .
$$




\section{OFFSET CONTINUATION IN THE LOG-STRETCH DOMAIN}

The log-stretch transform, proposed by Bolondi et al. (1982) and further developed by many other researchers, is a useful tool in DMO and OC processing. Applying a log-stretch transform of the form

$$
\sigma=\ln \left|\frac{t_{n}}{t_{*}}\right|,
$$

where $t_{*}$ is an arbitrarily chosen time constant, eliminates the time dependence of the coefficients in equation (1) and therefore makes this equation invariant to time shifts. After the double Fourier transform with respect to the midpoint coordinate $y$ and to the transformed (log-stretched) time coordinate $\sigma$, the partial differential equation (1) takes the form of an ordinary differential equation,

$$
h\left(\frac{d^{2} \widehat{\widehat{P}}}{d h^{2}}+k^{2} \widehat{\widehat{P}}\right)=i \Omega \frac{d \widehat{\widehat{P}}}{d h},
$$

where

$$
\widehat{\widehat{P}}(h)=\iint P\left(t_{n}=t_{*} \exp (\sigma), h, y\right) \exp (i \Omega \sigma-i k y) d \sigma d y .
$$

Equation (104) has the known general solution, expressed in terms of cylinder functions of complex order $\lambda=\frac{1+i \Omega}{2}$ (Watson, 1952)

$$
\widehat{\hat{P}}(h)=C_{1}(\lambda)(k h)^{\lambda} J_{-\lambda}(k h)+C_{2}(\lambda)(k h)^{\lambda} J_{\lambda}(k h),
$$

where $J_{-\lambda}$ and $J_{\lambda}$ are Bessel functions, and $C_{1}$ and $C_{2}$ stand for some arbitrary functions of $\lambda$ that do not depend on $k$ and $h$.

In the general case of offset continuation, $C_{1}$ and $C_{2}$ are constrained by the two initial conditions (62) and (63). In the special case of continuation from zero offset, we can neglect the second term in (106) as vanishing at the zero offset. The remaining term defines the following operator of inverse DMO in the $\Omega, k$ domain:

$$
\widehat{\hat{P}}(h)=\widehat{\widehat{P}}(0) Z_{\lambda}(k h),
$$

where $Z_{\lambda}$ is the analytic function

$$
\begin{aligned}
Z_{\lambda}(x) & =\Gamma(1-\lambda)\left(\frac{x}{2}\right)^{\lambda} J_{-\lambda}(x)={ }_{0} F_{1}\left(; 1-\lambda ;-\frac{x^{2}}{4}\right) \\
& =\sum_{n=0}^{\infty} \frac{(-1)^{n}}{n !} \frac{\Gamma(1-\lambda)}{\Gamma(n+1-\lambda)}\left(\frac{x}{2}\right)^{2 n},
\end{aligned}
$$

$\Gamma$ is the gamma function and ${ }_{0} F_{1}$ is the confluent hypergeometric limit function (Petkovsek et al., 1996). 
The DMO operator now can be derived as the inversion of operator (107), which is a simple multiplication by $1 / Z_{\lambda}(k h)$. Therefore, offset continuation becomes a multiplication by $Z_{\lambda}\left(k h_{2}\right) / Z_{\lambda}\left(k h_{1}\right)$ (the cascade of two operators). This fact demonstrates an important advantage of moving to the log-stretch domain: both offset continuation and DMO are simple filter multiplications in the Fourier domain of the log-stretched time coordinate.

In order to compare operator (107) with the known versions of log-stretch DMO, we need to derive its asymptotic representation for high frequency $\Omega$. The required asymptotic expression follows directly from the definition of function $Z_{\lambda}$ in equation (108) and the known asymptotic representation for a Bessel function of high order (Watson, 1952):

$$
J_{\lambda}(\lambda z) \stackrel{\lambda \rightarrow \infty}{\approx} \frac{(\lambda z)^{\lambda} \exp \left(\lambda \sqrt{1-z^{2}}\right)}{e^{\lambda} \Gamma(\lambda+1)\left(1-z^{2}\right)^{1 / 4}\left\{1+\sqrt{1-z^{2}}\right\}^{\sqrt{1-z^{2}}}}
$$

Substituting approximation (109) into (108) and considering the high-frequency limit of the resultant expression yields

$$
Z_{\lambda}(k h) \approx\left\{\frac{1+\sqrt{1-\left(\frac{k h}{\lambda}\right)^{2}}}{2}\right\}^{\lambda} \frac{\exp \left(\lambda\left[1-\sqrt{1-\left(\frac{k h}{\lambda}\right)^{2}}\right]\right)}{\left(1-\left(\frac{k h}{\lambda}\right)^{2}\right)^{1 / 4}} \approx F(\epsilon) e^{i \Omega \psi(\epsilon)}
$$

where $\epsilon$ denotes the ratio $\frac{2 k h}{\Omega}$,

$$
F(\epsilon)=\sqrt{\frac{1+\sqrt{1+\epsilon^{2}}}{2 \sqrt{1+\epsilon^{2}}}} \exp \left(\frac{1-\sqrt{1+\epsilon^{2}}}{2}\right)
$$

and

$$
\psi(\epsilon)=\frac{1}{2}\left(1-\sqrt{1+\epsilon^{2}}+\ln \left(\frac{1+\sqrt{1+\epsilon^{2}}}{2}\right)\right) .
$$

The asymptotic representation (110) is valid for high frequency $\Omega$ and $|\epsilon| \leq 1$. The phase function $\psi$ defined in (112) coincides precisely with the analogous term in Liner's exact log DMO (Liner, 1990), which provides the correct geometric properties of DMO. Similar expressions for the log-stretch phase factor $\psi$ were derived in different ways by Zhou et al. (1996) and Canning and Gardner (1996). However, the amplitude term $F(\epsilon)$ differs from the previously published ones because of the difference in the amplitude preservation properties.

A number of approximate log DMO operators have been proposed in the literature. As shown by Liner (1990), all of them but exact log DMO distort the geometry of reflection effects at large offsets. The distortion is caused by the implied approximations of the true phase function $\psi$. Bolondi's OC operator (Bolondi 
et al., 1982) implies $\psi(\epsilon) \approx-\frac{\epsilon^{2}}{8}$, Notfors' DMO (Notfors and Godfrey, 1987) implies $\psi(\epsilon) \approx 1-\sqrt{1+(\epsilon / 2)^{2}}$, and the "full DMO" (Bale and Jakubowicz, 1987) has $\psi(\epsilon) \approx \frac{1}{2} \ln \left[1-(\epsilon / 2)^{2}\right]$. All these approximations are valid for small $\epsilon$ (small offsets or small reflector dips) and have errors of the order of $\epsilon^{4}$ (Figure 6). The range of validity of Bolondi's operator is defined in equation (22).

Figure 6: Phase functions of the $\log$ DMO operators. Solid line: exact log DMO; dashed line: Bolondi's OC; dashed-dotted line: Bale's full DMO; dotted line: Notfors' DMO.

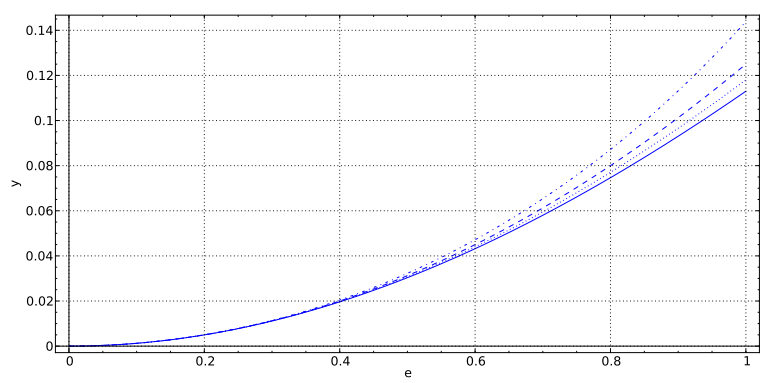

In practice, seismic data are often irregularly sampled in space but regularly sampled in time. This makes it attractive to apply offset continuation and DMO operators in the $\{\Omega, y\}$ domain, where the frequency $\Omega$ corresponds to the log-stretched time and $y$ is the midpoint coordinate. Performing the inverse Fourier transform on the spatial frequency transforms the inverse DMO operator (107) to the $\{\Omega, y\}$ domain, where the filter multiplication becomes a convolutional operator:

$$
\widehat{P}(\Omega, h, y)=\frac{\widehat{F}(\Omega)}{\sqrt{2 \pi}} \int_{|\xi|<h} \frac{h}{h^{2}-\xi^{2}} \widehat{P_{0}}(\Omega, y-\xi) \exp \left(-\frac{i \Omega}{2} \ln \left(1-\frac{\xi^{2}}{h_{1}^{2}}\right)\right) d \xi .
$$

Here $\widehat{F}(\Omega)$ is a high-pass frequency filter:

$$
\widehat{F}(\Omega)=\frac{\Gamma(1 / 2-i \Omega / 2)}{\sqrt{1 / 2} \Gamma(-i \Omega / 2)} .
$$

At high frequencies $\widehat{F}(\Omega)$ is approximately equal to $(-i \Omega)^{1 / 2}$, which corresponds to the half-derivative operator $\left(\frac{\partial}{\partial \sigma}\right)^{1 / 2}$, which, in turn, is equal to the $\left(t_{n} \frac{\partial}{\partial t_{n}}\right)^{1 / 2}$ term of the asymptotic OC operator (69). The difference between the exact filter $\widehat{F}$ and its approximation by the half-order derivative operator is shown in Figure 7 . This difference is a measure of the validity of asymptotic OC operators.

Inverting operator (113), we can obtain the DMO operator in the $\{\Omega, y\}$ domain.

\section{DISCUSSION}

The differential model for offset continuation is based on several assumptions. It is important to fully realize them in order to understand the practical limitations of this model. 

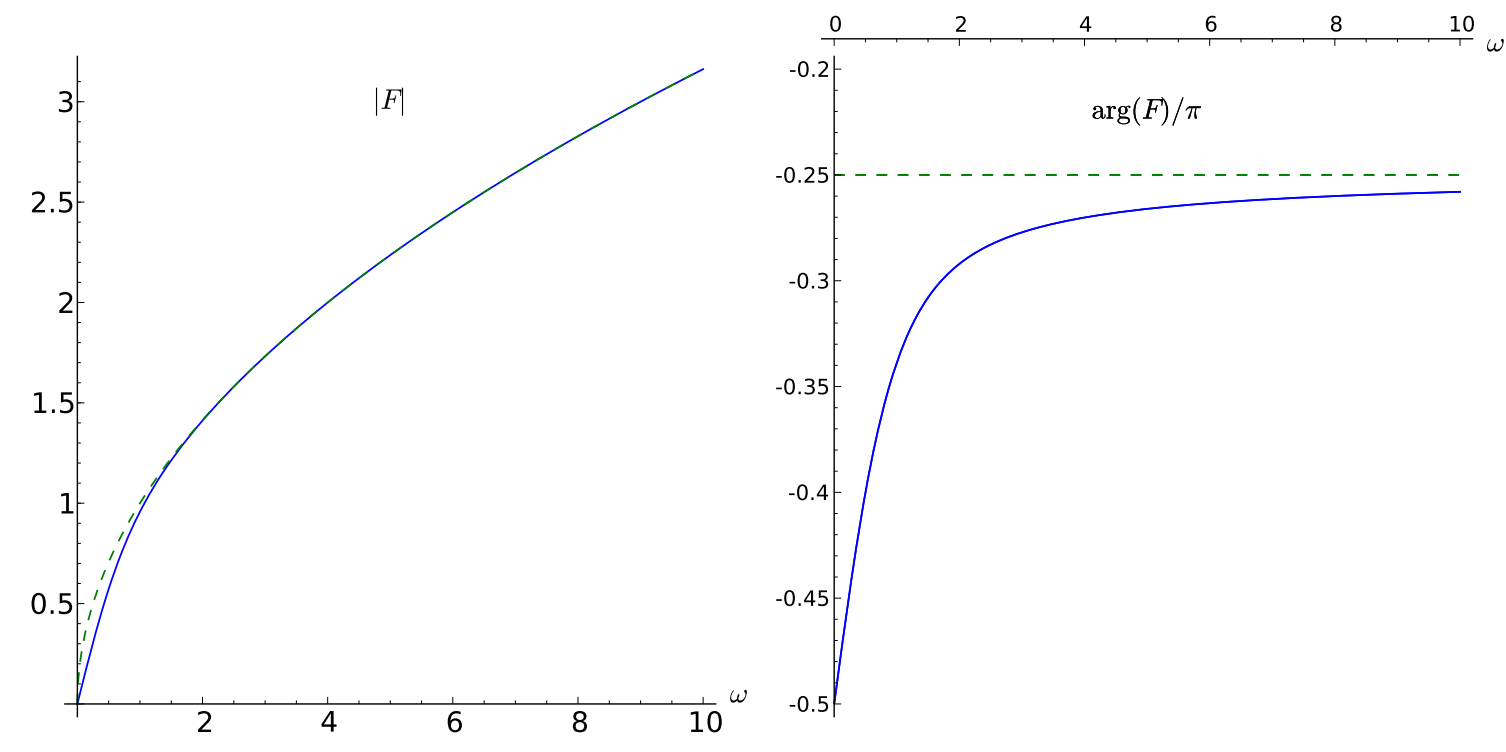

Figure 7: Amplitude (left) and phase (right) of the time filter in the log-stretch domain. The solid line is for the exact filter; the dashed line for its approximation by the half-order derivative filter. The horizontal axis corresponds to the dimensionless log-stretch frequency $\Omega$.

- The constant velocity assumption is essential for theoretical derivations. In practice, this limitation is not too critical, because the operators act locally. DMO and offset continuation algorithms based on the constant-velocity assumptions are widely used in practice (Hale, 1995).

- The single-mode assumption does not include multiple reflections in the model. If multiple events (with different apparent velocities) are present in the data, they might require extending the model. Convolving two (or more) differential offset continuation operators, corresponding to different velocities, we can obtain a higher-order differential operator for predicting multiple events.

- The continuous AVO assumption implies that the reflectivity variation with offset is continuous and can be neglected in a local neighborhood of a particular offset. While the offset continuation model correctly predicts the geometric spreading effects in the reflected wave amplitudes, it does not account for the variation of the reflection coefficient with offset.

- The 2.5-D assumption was implicit in the derivation of the offset continuation equation. According to this assumption, the reflector does not change in the cross-line direction, and we can always consider the reflection plane in two dimensions. 


\section{CONCLUSIONS}

I have introduced a partial differential equation (1) and proved that the process described by it provides for a kinematically and dynamically equivalent offset continuation transform. Kinematic equivalence means that in constant velocity media the reflection traveltimes are transformed to their true locations on different offsets. Dynamic equivalence means that, in the OC process, the geometric spreading term in the amplitudes of reflected waves transforms in accordance with the laws of geometric seismics, while the angle-dependent reflection coefficient stays the same.

The offset continuation equation can be applied directly to design OC operators of the finite-difference type. To construct integral OC operators, an initial value problem is solved for the offset continuation equation (1). For the special cases of continuation to zero offset (DMO) and continuation from zero offset (inverse DMO), the OC operators are related to the known forms of DMO operators: Hale's Fourier DMO, Born DMO, and Liner's "exact log DMO." The discovery of these relations sheds additional light on the problem of amplitude preservation in DMO.

\section{ACKNOWLEDGMENTS}

Sergey Goldin drew my attention to the role of curvature dependence in reflected wave amplitudes (Goldin and Fomel, 1995). I also acknowledge the support of Alexey Stovas, who introduced me to the world of DMO and collaborated with me on developing the asymptotic theory of offset continuation (Stovas and Fomel, 1996). Norman Bleistein collaborated with me on confirming the amplitude validity of offset continuation (Fomel et al., 1996; Fomel and Bleistein, 2001). Shuki Ronen and Richard Bale pointed out the importance of the log-stretch domain in DMO and related problems. I appreciate the encouragement of Jon Claerbout and Fabio Rocca regarding the analysis of the offset-continuation partial differential equation.

The financial support for this work was provided by the sponsors of the Stanford Exploration Project.

\section{REFERENCES}

Babich, V. M., 1991, Short-wavelength diffraction theory: asymptotic methods: Springer-Verlag.

Bagaini, C., and U. Spagnolini, 1993, Common shot velocity analysis by shot continuation operator: 63rd Ann. Internat. Mtg, Soc. of Expl. Geophys., 673-676.

- , 1996, 2-D continuation operators and their applications: Geophysics, 61, 18461858.

Bale, R., and H. Jakubowicz, 1987, Post-stack prestack migration: 57th Ann. Internat. Mtg, Soc. of Expl. Geophys., Session:S14.1. 
Beylkin, G., 1985, Imaging of discontinuities in the inverse scattering problem by inversion of a causal generalized Radon transform: Journal of Mathematical Physics, 26, 99-108.

Biondi, B., and N. Chemingui, 1994, Transformation of 3-D prestack data by azimuth moveout (AmO): 64th Ann. Internat. Mtg, Soc. of Expl. Geophys., 1541-1544.

Biondi, B., S. Fomel, and N. Chemingui, 1998, Azimuth moveout for 3-D prestack imaging: Geophysics, 63, 574-588.

Black, J. L., K. L. Schleicher, and L. Zhang, 1993, True-amplitude imaging and dip moveout: Geophysics, 58, 47-66.

Bleistein, N., 1984, Mathematical methods for wave phenomena: Academic Press Inc. (Harcourt Brace Jovanovich Publishers).

—, 1990, Born DMO revisited, in 60th Annual Internat. Mtg., Soc. Expl. Geophys., Expanded Abstracts: Soc. Expl. Geophys., 1366-1369.

Bleistein, N., and H. H. Jaramillo, 2000, A platform for Kirchhoff data mapping in sealer models of data acquisition: Geophys. Prosp., 48, 135-162.

Bleistein, N., J. W. Stockwell, and J. K. Cohen, 2001, Mathematics of multidimensional seismic imaging, migration, and inversion: Springer Verlag.

Bolondi, G., E. Loinger, and F. Rocca, 1982, Offset continuation of seismic sections: Geophys. Prosp., 30, 813-828.

Canning, A., and G. H. F. Gardner, 1996, Regularizing 3-D data sets with DmO: Geophysics, 61, 1103-1114. (Discussion and reply in GEO-62-4-1331).

Chemingui, N., and B. Biondi, 1996, Handling the irregular geometry in wide-azimuth surveys: 66th Ann. Internat. Mtg, Soc. of Expl. Geophys., 32-35.

Claerbout, J. F., 1976, Fundamentals of geophysical data processing: Blackwell.

Courant, R., 1962, Methods of mathematical physics: Interscience Publishers.

Deregowski, S. M., and F. Rocca, 1981, Geometrical optics and wave theory of constant offset sections in layered media: Geophys. Prosp., 29, 374-406.

Fomel, S., 2001, Three-dimensional seismic data regularization: PhD thesis, Stanford University.

_ 2003a, Asymptotic pseudounitary stacking operators: Geophysics, 68, 10321042.

— $2003 \mathrm{~b}$, Seismic reflection data interpolation with differential offset and shot continuation: Geophysics, 68, 733-744.

Fomel, S., and N. Bleistein, 2001, Amplitude preservation for offset continuation: Confirmation for Kirchhoff data: Journal of Seismic Exploration, 10, 121-130.

Fomel, S., N. Bleistein, H. Jaramillo, and J. K. Cohen, 1996, True amplitude DmO, offset continuation and $\mathrm{AvA} / \mathrm{AvO}$ for curved reflectors: 66th Ann. Internat. Mtg, Soc. of Expl. Geophys., 1731-1734.

Fomel, S. B., 1994, Kinematically equivalent differential operator for offset continuation of seismic sections: Russian Geology and Geophysics, 35, 122-134.

Fomel, S. B., and B. L. Biondi, 1995, The time and space formulation of azimuth moveout: 65th Ann. Internat. Mtg, Soc. of Expl. Geophys., 1449-1452.

Gazdag, J., 1978, Wave equation migration with the phase-shift method: Geophysics, 43, 1342-1351.

Goldin, S., 1990, A geometric approach to seismic processing: the method of discon- 
tinuities, in SEP-67: Stanford Exploration Project, 171-210.

Goldin, S. V., 1988, Transformation and recovery of discontinuities in problems of tomographic type: Institute of Geology and Geophysics.

— , 1994, Superposition and continuation of tranformations used in seismic migration: Russian Geology and Geophysics, 35, 131-145.

Goldin, S. V., and S. B. Fomel, 1995, Estimation of reflection coefficient in DMO: Russian Geology and Geophysics, 36, 103-115.

Gradshtein, I. S., and I. M. Ryzhik, 1994, Table of integrals, series, and products: Boston: Academic Press.

Haddon, R. A. W., and P. W. Buchen, 1981, Use of Kirchhoff's formula for body wave calculations in the earth: Geophys. J. Roy. Astr. Soc., 67, 587-598.

Hale, D., 1984, Dip-moveout by Fourier transform: Geophysics, 49, 741-757.

$\longrightarrow$, 1991, Course notes: Dip moveout processing: Soc. Expl. Geophys.

— , 1995, DMo Processing, in DMO processing: Soc. of Expl. Geophys., 496. (Reprints).

Hale, I. D., 1983, Dip moveout by Fourier transform: PhD thesis, Stanford University. Hill, S. J., R. Stolt, and S. Chiu, 2001, Altering offsets and azimuths: The Leading Edge, 20, 210-213.

Liner, C., 1990, General theory and comparative anatomy of dip moveout: Geophysics, 55, 595-607.

Liner, C. L., 1991, Born theory of wave-equation dip moveout: Geophysics, 56, 182189.

Liner, C. L., and J. K. Cohen, 1988, An amplitude-preserving inverse of hale's DmO: 58th Ann. Internat. Mtg, Soc. of Expl. Geophys., Session:S17.5.

Notfors, C. D., and R. J. Godfrey, 1987, Dip moveout in the frequency-wavenumber domain (short note): Geophysics, 52, 1718-1721.

Petkovsek, M., H. S. Wilf, and D. Zeilberger, 1996, $A=B$ : A K Peters Ltd.

Ronen, J., 1987, Wave equation trace interpolation: Geophysics, 52, 973-984.

Ronen, S., 1994, Handling irregular geometry: Equalized DmO and beyond: 64th Ann. Internat. Mtg, Soc. of Expl. Geophys., 1545-1548.

Ronen, S., V. Sorin, and R. Bale, 1991, Spatial dealiasing of 3-D seismic reflection data: Geophysical Journal International, 503-511.

Stolt, R. H., 1978, Migration by Fourier transform: Geophysics, 43, 23-48. (Discussion and reply in GEO-60-5-1583).

Stovas, A. M., and S. B. Fomel, 1996, Kinematically equivalent integral DMO operators: Russian Geology and Geophysics, 37, 102-113.

Tenenbaum, M., and H. Pollard, 1985, Ordinary differential equations : an elementary textbook for students of mathematics, engineering, and the sciences: Dover Publications.

Červený, V., 2001, Seismic ray theory: Cambridge University Press.

Watson, G. N., 1952, A treatise on the theory of Bessel functions, 2nd ed.: Cambridge University Press.

Zhou, B., I. M. Mason, and S. A. Greenhalgh, 1996, An accurate formulation of log-stretch dip moveout in the frequency-wavenumber domain: Geophysics, 61, 815-820. 


\section{APPENDIX A \\ SECOND-ORDER REFLECTION TRAVELTIME DERIVATIVES}

This appendix contains a derivation of equations connecting second-order partial derivatives of the reflection traveltime with the geometric properties of the reflector in a constant velocity medium. These equations are used in the main text of this paper to describe the amplitude behavior of offset continuation. Let $\tau(s, r)$ be the reflection traveltime from the source $s$ to the receiver $r$. Consider a formal equality

$$
\tau(s, r)=\tau_{1}(s, x(s, r))+\tau_{2}(x(s, r), r),
$$

where $x$ is the reflection point parameter, $\tau_{1}$ corresponds to the incident ray, and $\tau_{2}$ corresponds to the reflected ray. Differentiating (A-1) with respect to $s$ and $r$ yields

$$
\begin{aligned}
& \frac{\partial \tau}{\partial s}=\frac{\partial \tau_{1}}{\partial s}+\frac{\partial \tau}{\partial x} \frac{\partial x}{\partial s} \\
& \frac{\partial \tau}{\partial r}=\frac{\partial \tau_{2}}{\partial r}+\frac{\partial \tau}{\partial x} \frac{\partial x}{\partial r}
\end{aligned}
$$

According to Fermat's principle, the two-point reflection ray path must correspond to the traveltime stationary point. Therefore

$$
\frac{\partial \tau}{\partial x} \equiv 0
$$

for any $s$ and $r$. Taking into account (A-4) while differentiating (A-2) and (A-3), we get

$$
\begin{aligned}
\frac{\partial^{2} \tau}{\partial s^{2}} & =\frac{\partial^{2} \tau_{1}}{\partial s^{2}}+B_{1} \frac{\partial x}{\partial s} \\
\frac{\partial^{2} \tau}{\partial r^{2}} & =\frac{\partial^{2} \tau_{2}}{\partial r^{2}}+B_{2} \frac{\partial x}{\partial r} \\
\frac{\partial^{2} \tau}{\partial s \partial r} & =B_{1} \frac{\partial x}{\partial r}=B_{2} \frac{\partial x}{\partial s}
\end{aligned}
$$

where

$$
B_{1}=\frac{\partial^{2} \tau_{1}}{\partial s \partial x} ; B_{2}=\frac{\partial^{2} \tau_{2}}{\partial r \partial x} .
$$

Differentiating equation (A-4) gives us the additional pair of equations

$$
\begin{aligned}
& C \frac{\partial x}{\partial s}+B_{1}=0 \\
& C \frac{\partial x}{\partial r}+B_{2}=0
\end{aligned}
$$

where

$$
C=\frac{\partial^{2} \tau}{\partial x^{2}}=\frac{\partial^{2} \tau_{1}}{\partial x^{2}}+\frac{\partial^{2} \tau_{2}}{\partial x^{2}}
$$


Solving the system (A-8) - (A-9) for $\frac{\partial x}{\partial s}$ and $\frac{\partial x}{\partial r}$ and substituting the result into (A-5) - (A-7) produces the following set of expressions:

$$
\begin{aligned}
\frac{\partial^{2} \tau}{\partial s^{2}} & =\frac{\partial^{2} \tau_{1}}{\partial s^{2}}-C^{-1} B_{1}^{2} \\
\frac{\partial^{2} \tau}{\partial r^{2}} & =\frac{\partial^{2} \tau_{2}}{\partial r^{2}}-C^{-1} B_{2}^{2} \\
\frac{\partial^{2} \tau}{\partial s \partial r} & =-C^{-1} B_{1} B_{2} .
\end{aligned}
$$

In the case of a constant velocity medium, expressions (A-10) to (A-12) can be applied directly to the explicit equation for the two-point eikonal

$$
\tau_{1}(y, x)=\tau_{2}(x, y)=\frac{\sqrt{(x-y)^{2}+z^{2}(x)}}{v} .
$$

Differentiating (A-13) and taking into account the trigonometric relationships for the incident and reflected rays (Figure 1), one can evaluate all the quantities in (A-10) to (A-12) explicitly. After some heavy algebra, the resultant expressions for the traveltime derivatives take the form

$$
\begin{gathered}
\frac{\partial \tau}{\partial s}=\frac{\partial \tau_{1}}{\partial s}=\frac{\sin \alpha_{1}}{v} \quad ; \quad \frac{\partial \tau}{\partial r}=\frac{\partial \tau_{2}}{\partial r}=\frac{\sin \alpha_{2}}{v} ; \\
\frac{\partial \tau_{1}}{\partial x}=\frac{\sin \gamma}{v \cos \alpha} ; \quad \frac{\partial \tau_{2}}{\partial x}=-\frac{\sin \gamma}{v \cos \alpha} ; \\
B_{1}=\frac{\partial^{2} \tau_{1}}{\partial s \partial x}=\frac{\cos \alpha_{1}}{v D \cos \alpha}\left(-1-\frac{\sin \gamma}{\cos \alpha} \sin \alpha_{1}\right) ; \\
B_{2}=\frac{\partial^{2} \tau_{2}}{\partial r \partial x}=\frac{\cos \alpha_{2}}{v D \cos \alpha}\left(-1+\frac{\sin \gamma}{\cos \alpha} \sin \alpha_{2}\right) ; \\
B_{1} B_{2}=\frac{\cos ^{6} \gamma}{v^{2} D^{2} a^{4}} ; B_{1}+B_{2}=-2 \frac{\cos ^{3} \gamma}{v D a^{2}}\left(2 a^{2}-1\right) ; \\
\frac{\partial^{2} \tau_{1}}{\partial x^{2}}=\frac{\cos ^{2} \gamma+D K}{v D \cos ^{3} \alpha} \cos \alpha_{1} ; \frac{\partial^{2} \tau_{2}}{\partial x^{2}}=\frac{\cos ^{2} \gamma+D K}{v D \cos ^{3} \alpha} \cos \alpha_{2} ; \\
C=\frac{\partial^{2} \tau_{1}}{\partial x^{2}}+\frac{\partial^{2} \tau_{2}}{\partial x^{2}}=2 \cos \gamma \frac{\cos ^{2} \gamma+D K}{v D \cos ^{3} \alpha} .
\end{gathered}
$$

Here $D$ is the length of the normal (central) ray, $\alpha$ is its dip angle $\left(\alpha=\frac{\alpha_{1}+\alpha_{2}}{2}\right.$, $\left.\tan \alpha=z^{\prime}(x)\right), \gamma$ is the reflection angle $\left(\gamma=\frac{\alpha_{2}-\alpha_{1}}{2}\right), K$ is the reflector curvature at the reflection point $\left(K=z^{\prime \prime}(x) \cos ^{3} \alpha\right)$, and $a$ is the dimensionless function of $\alpha$ and $\gamma$ defined in (47).

The equations derived in this appendix were used to obtain the equation

$$
\tau_{n}\left(\frac{\partial^{2} \tau_{n}}{\partial y^{2}}-\frac{\partial^{2} \tau_{n}}{\partial h^{2}}\right)=4\left(\tau \frac{\partial^{2} \tau}{\partial s \partial r}+\frac{\cos ^{2} \gamma}{v^{2}}\right)=4 \frac{\cos ^{2} \gamma}{v^{2}}\left(\frac{\sin ^{2} \alpha+D K}{\cos ^{2} \gamma+D K}\right)
$$

which coincides with (50) in the main text. 


\section{APPENDIX B}

\section{THE KINEMATICS OF OFFSET CONTINUATION}

This Appendix presents an alternative method to derive equation (70), which describes the summation path of the integral offset continuation operator. The method is based on the following considerations.

The summation path of an integral (stacking) operator coincides with the phase function of the impulse response of the inverse operator. Impulse response is by definition the operator reaction to an impulse in the input data. For the case of offset continuation, the input is a reflection common-offset gather. From the physical point of view, an impulse in this type of data corresponds to the special focusing reflector (elliptical isochrone) at the depth. Therefore, reflection from this reflector at a different constant offset corresponds to the impulse response of the OC operator. In other words, we can view offset continuation as the result of cascading prestack commonoffset migration, which produces the elliptic surface, and common-offset modeling (inverse migration) for different offsets. This approach resemble that of Deregowski and Rocca (1981). It was also applied to a more general case of azimuth moveout (AMO) by Fomel and Biondi (1995) and fully generalized by Bleistein and Jaramillo (2000). The geometric approach implies that in order to find the summation pass of the OC operator, one should solve the kinematic problem of reflection from an elliptic reflector whose focuses are in the shot and receiver locations of the output seismic gather.

In order to solve this problem, let us consider an elliptic surface of the general form

$$
h(x)=\sqrt{d^{2}-\beta\left(x-x^{\prime}\right)^{2}},
$$

where $0<\beta<1$. In a constant velocity medium, the reflection ray path for a given source-receiver pair on the surface is controlled by the position of the reflection point $x$. Fermat's principle provides a required constraint for finding this position. According to Fermat's principle, the reflection ray path corresponds to a stationary value of the travel-time. Therefore, in the neighborhood of this path,

$$
\frac{\partial \tau(s, r, x)}{\partial x}=0
$$

where $s$ and $r$ stand for the source and receiver locations on the surface, and $\tau$ is the reflection traveltime

$$
\tau(s, r, x)=\frac{\sqrt{h^{2}(x)+(s-x)^{2}}}{v}+\frac{\sqrt{h^{2}(x)+(r-x)^{2}}}{v} .
$$

Substituting equations (B-3) and (B-1) into (B-2) leads to a quadratic algebraic equation on the reflection point parameter $x$. This equation has the explicit solution

$$
x(s, r)=x^{\prime}+\frac{\xi^{2}+H^{2}-h^{2}+\operatorname{sign}\left(h^{2}-H^{2}\right) \sqrt{\left(\xi^{2}-H^{2}-h^{2}\right)^{2}-4 H^{2} h^{2}}}{2 \xi(1-\beta)},
$$


where $h=(r-s) / 2, \xi=y-x^{\prime}, y=(s+r) / 2$, and $H^{2}=d^{2}\left(\frac{1}{\beta}-1\right)$. Replacing $x$ in equation (B-3) with its expression (B-4) solves the kinematic part of the problem, producing the explicit traveltime expression

$$
\tau(s, r)=\left\{\begin{array}{ll}
\frac{1}{v} \sqrt{\frac{4 h^{2}-\beta(f+g)^{2}}{1-\beta}} & \text { for } h^{2}>H^{2} \\
\frac{1}{v} \sqrt{\frac{4 h^{2}+\beta(F+G)^{2}}{1-\beta}} & \text { for } h^{2}<H^{2}
\end{array},\right.
$$

where

$$
\begin{array}{lll}
f=\sqrt{\left(r-x^{\prime}\right)^{2}-H^{2}} & , \quad g=\sqrt{\left(s-x^{\prime}\right)^{2}-H^{2}} \\
F=\sqrt{H^{2}-\left(r-x^{\prime}\right)^{2}} & , \quad G=\sqrt{H^{2}-\left(s-x^{\prime}\right)^{2}} .
\end{array}
$$

The two branches of equation (B-5) correspond to the difference in the geometry of the reflected rays in two different situations. When a source-and-receiver pair is inside the focuses of the elliptic reflector, the midpoint $y$ and the reflection point $x$ are on the same side of the ellipse with respect to its small semi-axis. They are on different sides in the opposite case (Figure B-1).

Figure B-1: .

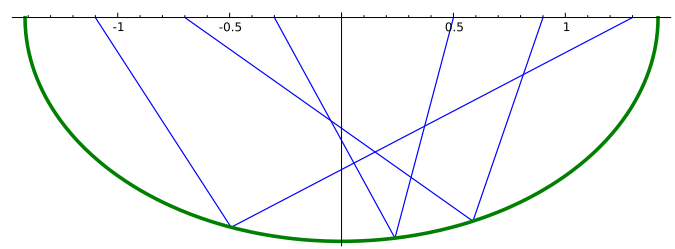

Reflections from an ellipse. The three pairs of reflected rays correspond to a common midpoint (at 0.1) and different offsets. The focuses of the ellipse are at 1 and -1 .

If we apply the NMO correction, equation (B-5) is transformed to

$$
\tau_{n}(s, r)=\left\{\begin{array}{ll}
\frac{1}{v} \sqrt{\frac{\beta}{1-\beta}} \sqrt{4 h^{2}-(f+g)^{2}} & \text { for } h^{2}>H^{2} \\
\frac{1}{v} \sqrt{\frac{\beta}{1-\beta}} \sqrt{4 h^{2}+(F+G)^{2}} & \text { for } h^{2}<H^{2}
\end{array} .\right.
$$

Then, recalling the relationships between the parameters of the focusing ellipse $r, x^{\prime}$ and $\beta$ and the parameters of the output seismic gather (Deregowski and Rocca, 1981)

$$
r=\frac{v t_{n}}{2}, x^{\prime}=y, \beta=\frac{t_{n}^{2}}{t_{n}^{2}+\frac{4 h^{2}}{v^{2}}}, H=h,
$$


and substituting expressions (B-7) into equation (B-6) yields the expression

$$
t_{1}\left(s_{1}, r_{1} ; s, r, t_{n}\right)=\left\{\begin{array}{ll}
\frac{t_{n}}{2 h} \sqrt{4 h_{1}^{2}-(f+g)^{2}} & \text { for } h_{1}^{2}>h^{2} \\
\frac{t_{2}}{2 h} \sqrt{4 h_{1}^{2}+(F+G)^{2}} & \text { for } h_{1}^{2}<h^{2}
\end{array},\right.
$$

where

$$
\begin{aligned}
f & =\sqrt{\left(r_{1}-r\right)\left(r_{1}-s\right)}, g=\sqrt{\left(s_{1}-r\right)\left(s_{1}-s\right)} \\
F & =\sqrt{\left(r-r_{1}\right)\left(r_{1}-s\right)}, G=\sqrt{\left(s_{1}-r\right)\left(s-s_{1}\right)}
\end{aligned}
$$

It is easy to verify algebraically the mathematical equivalence of equation (B-8) and equation (70) in the main text. The kinematic approach described in this appendix applies equally well to different acquisition configurations of the input and output data. The source-receiver parameterization used in (B-8) is the actual definition for the summation path of the integral shot continuation operator (Bagaini and Spagnolini, 1993, 1996). A family of these summation curves is shown in Figure B-2.

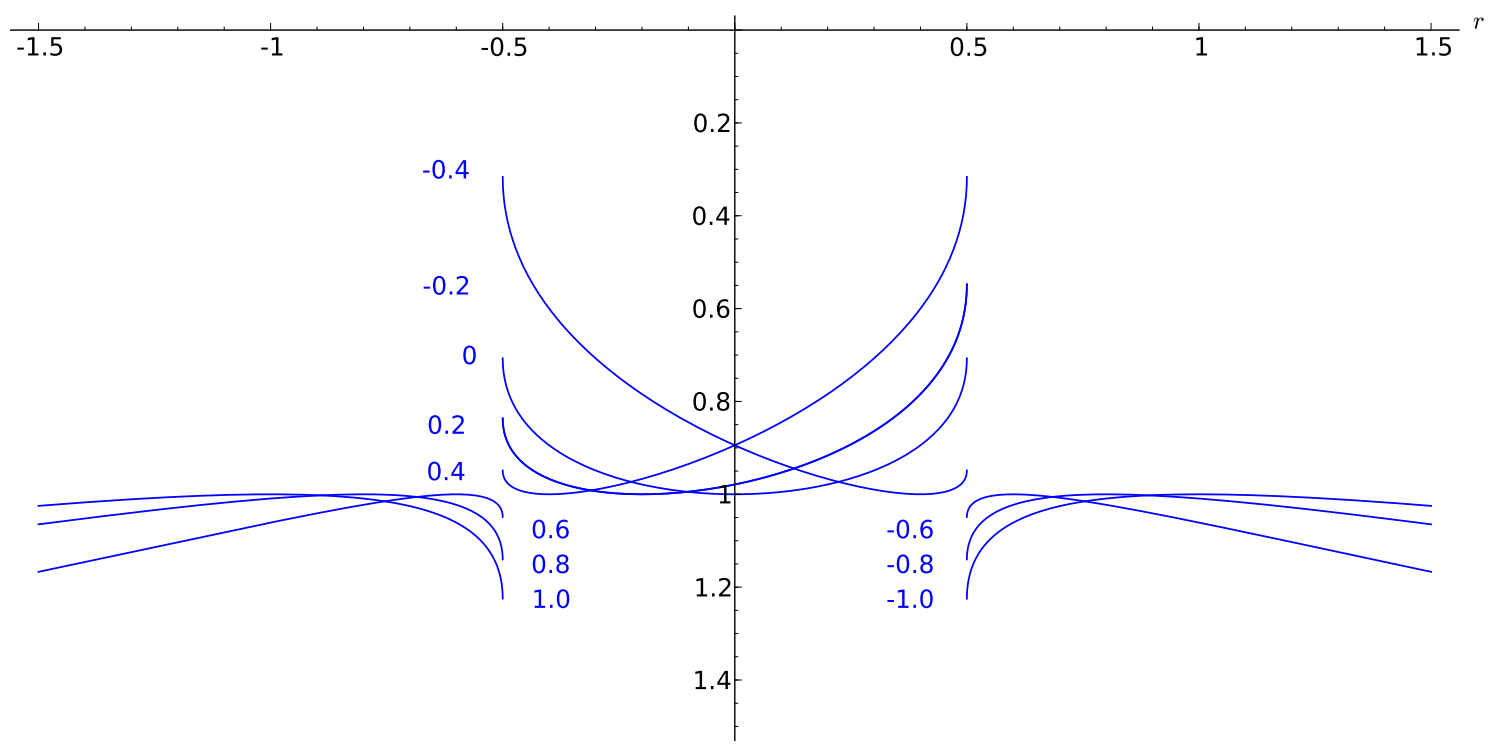

Figure B-2: .

Summation paths of the integral shot continuation. The output source is at -0.5 $\mathrm{km}$. The output receiver is at $0.5 \mathrm{~km}$. The indexes of the curves correspond to the input source location. 